\title{
Congestion probabilities in CDMA-based networks supporting batched Poisson traffic
}

\begin{abstract}
I. D. Moscholios ${ }^{1}$, G. A. Kallos ${ }^{2}$, V. G. Vassilakis ${ }^{3}$ and M. D. Logothetis ${ }^{4}$
1. Dept. of Informatics \& Telecommunications, University of Peloponnese, 22100 Tripolis, Greece 2. BT Technology, Service and Operations, IP5 3RE Ipswich, U.K.

3. Dept. of Electronic Engineering, University of Surrey, GU2 7XH Guilford, U.K.

4. WCL, Dept. of Electrical \& Computer Engineering, University of Patras, 26504 Patras, Greece.

E-mails: idm@uop.gr, george.kallos@bt.com, v.vasilakis@surrey.ac.uk, mlogo@upatras.gr

Abstract-We propose a new multirate teletraffic loss model for the calculation of time and call congestion probabilities in CDMA-based networks that accommodate calls of different serviceclasses whose arrival follows a batched Poisson process. The latter is more "peaked" and "bursty" than the ordinary Poisson process. The acceptance of calls in the system is based on the partial batch blocking discipline. This policy accepts a part of the batch (one or more calls) and discards the rest if the available resources are not enough to accept the whole batch. The proposed model takes into account the multiple access interference, the notion of local (soft) blocking, user's activity and the interference cancellation. Although the analysis of the model does not lead to a product form solution of the steady state probabilities, we show that the calculation of the call-level performance metrics, time and call congestion probabilities, can be based on approximate but recursive formulas. The accuracy of the proposed formulas are verified through simulation and found to be quite satisfactory.
\end{abstract}

Keywords - CDMA; time-call congestion; batched Poisson; recursive formula; call admission.

\section{Introduction}

The CDMA (Code-Division Multiple Access) is a family of channel access techniques used by various radio communication technologies [1]. For example the Wideband CDMA (W-CDMA) and the Time Division CDMA are used in the third generation (3G) mobile technology standards (UMTS, CDMA2000) [2]. Furthermore, schemes such as the Multi-Carrier CDMA [3] and the Large Area Synchronised CDMA [4] have been considered by some of the fourth generation (4G) networks [5], [6]. CDMA-based networks are known for efficient frequency spectrum utilization, good signal quality, and enhanced security, just to name a few. Recently there started some efforts in developing concepts for future fifth generation (5G) networks. Some of them envision CDMA technology as a key multiple access method [7]. While nothing is clear yet with regard to the future 5G networks, it's very likely that they would integrate different current existing and future wireless network technologies (including CDMA-based) to ensure seamless roaming between various technology standards. 
CDMA networks are an exemplary case where call admission control is subject to the levels of the interference experienced from the Base Station in the air medium. In the uplink direction, users belonging to a cell are not only sharing the same bandwidth spectrum among them, but also with users of the neighboring cells. Such interference based networks implement different call admission control policies with respect to traditional wire networks that take into account the aforementioned multiple access interference. The application of traditional performance analysis methods is therefore challenging and necessitates adaptation, especially when the heterogeneous nature of the traffic is considered.

Herein, we consider a CDMA reference cell that supports calls from $K$ different serviceclasses and examine the uplink direction (from a mobile user to the base station that controls the cell). The reference cell is modeled as a multirate loss system whose capacity consists of a fixed and integer number of channels. Similarly, the radio resource requirements of each call correspond to an integer number of channels. A new service-class $k(k=1, \ldots, K)$ call is accepted in the cell if the requested number of channels is available. More precisely, the admission of a new call is based on the estimation of the total interference increase (own-cell and other-cell interference plus thermal noise) caused by the new call's acceptance. An accepted call remains in the system for an exponentially distributed service time. Due to the existence of interferences, soft or local blocking occurs. That is, a new call can be blocked and lost in any system state if its acceptance increases the experienced noise of all in-service calls above a tolerable level, given that, according to the CDMA principle, a call is noise for all other calls. This call admission policy corresponds to the complete sharing (CS) policy in wire networks, whereby all calls compete for all bandwidth resources [8].

The previous model of a CDMA cell has been adopted in [9]-[12]. In these papers, the formulas proposed for the calculation of call blocking probabilities (CBP) in the cell, resemble the classical Kaufman-Roberts formula used for the CBP calculation in the Erlang Multirate Loss Model (EMLM). The EMLM refers to a single link of certain capacity that accommodates, under the CS policy, Poisson arriving calls of different service-classes with different bandwidth requirements and generally distributed service times [13], [14]. More precisely, in [9] and [11] an extension of the EMLM is considered which is based on the Delbrouck's model [15]. The latter generalizes the EMLM since it allows the call-arrival process to have different peakedness factors ${ }^{1}$. In [10], calls arrive in the cell according to a Poisson process. In [12], calls come from a finite number of sources, a case that can be realistic especially in cells that have a limited coverage area. Apart from the different call arrival processes considered in [9]-[12], there is also another difference in the way the notion of local blocking is modelled. In [9] (also adopted in [11]), this modelling is more complicated compared to the one proposed in [10] (also adopted in [12]). In subsection 3.2, we compare the two approaches and adopt the model of [10] as it is more realistic for CDMAbased systems.

\footnotetext{
${ }^{1}$ The peakedness factor $z$ is the ratio of the variance over the mean of the number of arrivals; if $z=1$, the arrival process is Poisson; if $z<1$, the arrival process is quasi-random; if $z>1$, the process is more peaked and bursty than Poisson (e.g. overflow traffic).
} 
In this paper, we consider the abovementioned model of a CDMA reference cell and analyze the case of batched Poisson call arrivals. In a batched Poisson arrival process, which is more bursty than a Poisson process, batches of calls occur at time-points which follow the negative exponential distribution. New calls are accepted in the cell according to the partial batch blocking discipline. The latter assumes that a part of the entire batch (one or more calls) can be accepted in the cell while the rest of it is discarded, when the available resources are not enough to accommodate the entire batch. As far as the distribution of the batch size is concerned, we consider a general distribution. In the case of the geometric batch size distribution (a memoryless distribution and a discrete equivalent of the exponential distribution), the proposed model coincides with the model of [9], [11]. The steady-state probabilities in the proposed model do not have a product form solution. However, we propose approximate but recursive formulas for the calculation of time and call congestion probabilities (TC and CC probabilities, respectively). The analytical results are verified by simulation and found to be absolutely satisfactory. Note at this point that TC probabilities are determined by the proportion of time the system is congested. An observer, who is not part of the system, can measure this probability. On the other hand, CC probabilities are determined by the proportion of arriving calls that find the system congested. An observer who is part of the system (i.e. an arriving call) can measure this probability. These probabilities coincide in the case of Poisson arrivals due to the Poisson Arrivals See Time Averages (PASTA) property [8]. In the case of a batched Poisson process, CC probabilities are higher than TC probabilities.

To the best of our knowledge, a call-level analysis in CDMA networks under a batched Poisson process has not been considered before. On the other hand, many papers study this arrival process (at call level) in wired networks (see e.g., [16]-[20]). In [16], the EMLM is extended to include a batched Poisson process under the geometric batch size distribution and the partial batch blocking discipline. In [17], a general batch size distribution is considered. Herein, we name the model of [17], Batch Poisson EMLM (BP-EMLM). In [18], the BPEMLM is extended to include the bandwidth reservation policy. In this policy, a part of the available bandwidth is reserved to benefit calls with high bandwidth requirements. In [19][20], the BP-EMLM is extended to include the case where in-service calls can compress their bandwidth during their lifetime in the system. In the proposed model, we assume that inservice calls do not compress their bandwidth.

In addition to the batched Poisson process, we study the effect of interference cancellation on TC and CC probabilities. Interference cancellation receivers reduce only the own-cell interference and not the other-cell interference or the thermal noise [21]. This reduction results in the decrease of TC and CC probabilities in the proposed model. Note that the introduction of the interference cancellation in the call-level modelling of CDMA networks has been considered only in [12], where calls come from a finite number of sources.

This paper is organized as follows: In section 2, we present the basic relations in the uplink direction of a CDMA reference cell. In subsection 2.1, we review the relations for the total received power of a service-class $k$ call with or without the existence of interference cancellation. In subsection 2.2, we propose a formula for the determination of the maximum 
number of service-class $k$ calls in the cell under the existence of interference cancellation. In subsection 2.3, we calculate the uplink capacity and the bandwidth requirement of serviceclass $k$ calls. In section 3, we consider the case of Poisson arrivals and calculate CBP in the case of hard blocking ${ }^{2}$ only (subsection 3.1) and when hard and soft blocking co-exist (subsection 3.2). In section 4, we consider the case of batched Poisson arrivals and calculate TC and CC probabilities in the case of hard blocking only (subsection 4.1) and when hard and soft blocking co-exist (subsection 4.2). In both subsections 3.2 and 4.2 we also consider the case of interference cancellation. In section 5, we present numerical results and evaluate the proposed formulas by comparing analytical with simulation results. We conclude in section 6 . In the Appendix, we provide the proof for the formula of the maximum number of serviceclass $k$ calls in the cell under the existence of interference cancellation.

\section{Basic relations in the uplink direction of a CDMA reference cell}

\subsection{Determination of the total received power of a service-class $\boldsymbol{k}$ call}

Consider a CDMA reference cell controlled by a base station and surrounded by other neighbouring cells. In the reference cell, we examine the uplink direction and model the reference cell as a multirate loss model. The cell accommodates calls of $K$ different serviceclasses. A service-class $k$ call $(k=1, \ldots, K)$ alternates between periods of transmission (active periods) and periods of non-transmission (passive periods). The ratio of “active periods” over "active + passive periods" is the activity factor of a service-class $k$ call, $v_{k}$, where $v_{k} \leq 1$. Typical values of $v_{k}$, for dimensioning purposes, are: $v_{k}=1.0$ if $k$ is a data service-class and $v_{k}$ $=0.67$ if $k$ is a voice service-class (see pp. 187, Table 8.9 in [2]).

In CDMA systems, all users transmit within the same frequency band which means that a single user "sees" the signals generated by all other users as interference. In that case, the base station's capacity in the CDMA reference cell is limited by the so called Multiple Access Interference (MAI) [2]. The latter consists of two types of interference: 1) the own-cell interference, $P_{\text {own }}$, which is caused by the mobile users of the reference cell and 2) the othercell interference, $P_{\text {other, }}$, which refers to the interference power received from the mobile users of the neighbouring cells. Since the MAI has a stochastic nature, we speak about the soft capacity (or the interference limited capacity) of the radio interface (see also pp. 225 in [22]). Apart from $P_{\text {own }}$ and $P_{\text {other }}$ we take into account the existence of thermal noise, $P_{\text {noise }}$, which corresponds to the interference of an empty CDMA system. A typical value of the thermal noise power density, in W-CDMA systems, is $174 \mathrm{dBm} / \mathrm{Hz}$ (see pp. 178 in [2]).

The values of $P_{\text {own }}$ can be reduced by the so called interference cancellation; the latter is not effective towards $P_{\text {other }}$ and $P_{\text {noise }}$. The interference cancellation efficiency is denoted by $\beta$ and defined by the ratio [21]:

$$
\beta=\frac{P_{o w n}^{N O I C}-P_{o w n}}{P_{o w n}^{N O I C}} \Rightarrow P_{o w n}=P_{o w n}^{N O I C}(1-\beta)
$$

\footnotetext{
${ }^{2}$ Hard blocking occurs when the bandwidth requirement of a new call is higher than the available resources of the system. This type of blocking appears in wired networks.
} 
where $P_{o w n}^{\text {No IC }}$ is the own-cell interference without interference cancellation. In what follows, we assume that $\beta$ is constant $(0 \leq \beta<1)$ and common to all service-classes while it is independent of the specific receiver implementation and radio link conditions ([21], [23]).

Due to the existence of interference cancellation, and by denoting as $p_{k}$ the total received power from a service-class $k$ user, we can write the power control equation for service-class $k$ as follows [21]:

$\left(\frac{E_{b}}{N_{0}}\right)_{k}=G_{k} \frac{p_{k}}{\left(P_{\text {own }}-p_{k}\right)(1-\beta)+P_{\text {other }}+P_{\text {noise }}}$

where $\left(\frac{E_{b}}{N_{0}}\right)_{k}$ is the signal energy per bit divided by the noise spectral density, required to meet a predefined Block Error Rate, $G_{k}=\frac{W}{v_{k} R_{k}}$ is the processing gain of service-class $k$ in the uplink direction with user activity factor $v_{k}$, data rate $R_{k}$ and $W$ the chip rate of 3840 kcps (in W-CDMA systems).

Based on eq. (2), the values of $p_{k}$ can be obtained by:

$$
p_{k}=\left(\frac{E_{b}}{N_{0}}\right)_{k} \frac{\left(P_{\text {own }}(1-\beta)+P_{\text {other }}+P_{\text {noise }}\right)}{G_{k}+\left(\frac{E_{b}}{N_{0}}\right)_{k}(1-\beta)} \Rightarrow p_{k}=\frac{\left(P_{\text {own }}(1-\beta)+P_{\text {other }}+P_{\text {noise }}\right)}{1-\beta+\frac{G_{k}}{\left(\frac{E_{b}}{N_{0}}\right)_{k}}}
$$

\section{Note 1:}

If $\beta=0$ (i.e., interference cancellation is not considered) then eq. (2) and eq. (3) result in (see also pp. 181 in [2], eqs (8.4) and (8.5), respectively):

$$
\begin{aligned}
& \left(\frac{E_{b}}{N_{0}}\right)_{k}=G_{k} \frac{p_{k}}{P_{\text {total }}-p_{k}} \\
& p_{k}=\left(\frac{E_{b}}{N_{0}}\right)_{k} \frac{P_{\text {total }}}{G_{k}+\left(\frac{E_{b}}{N_{0}}\right)_{k}} \Rightarrow p_{k}=\frac{P_{\text {total }}}{1+\frac{G_{k}}{\left(\frac{E_{b}}{N_{0}}\right)_{k}}}
\end{aligned}
$$

where $P_{\text {total }}=P_{\text {own }}+P_{\text {other }}+P_{\text {noise }}$ is the total received power at the base station. 


\subsection{Determination of the maximum number of service-class $\boldsymbol{k}$ calls in the cell}

Let $N_{k}$ be the maximum number of service-class $k$ calls in the cell. Assuming that $P_{\text {own }}=p_{k} N_{k}$, we can calculate $P_{\text {own }}$, via eq. (3), as a function of $N_{k}{ }^{3}$ :

$$
P_{\text {own }}=\frac{N_{k}\left(P_{\text {other }}+P_{\text {noise }}\right)}{(1-\beta)-N_{k}(1-\beta)+\frac{G_{k}}{\left(\frac{E_{b}}{N_{0}}\right)_{k}}}
$$

Consider now the Noise Rise (NR) which is defined as the ratio of the total received power at the base station to the thermal noise power (see also pp. 227 in [22]):

$$
N R=\frac{P_{\text {total }}}{P_{\text {noise }}}=\frac{P_{\text {own }}+P_{\text {other }}+P_{\text {noise }}}{P_{\text {noise }}}
$$

The NR is related to the total uplink cell load, $\eta_{U L}$, according to the formula (see also pp. 182, eq. (8.9) in [2]):

$$
N R=\frac{1}{1-\eta_{U L}}
$$

where: $\eta_{U L}$ is defined as the ratio of the received power from all active users to the total received power, i.e., $\eta_{U L}=\frac{P_{o w n}+P_{o t h e r}}{P_{\text {total }}}$.

Based on eqs. (7) and (8) we have:

$$
\frac{P_{o w n}+P_{\text {other }}+P_{\text {noise }}}{P_{\text {noise }}}=\frac{1}{1-\eta_{U L}}
$$

Substituting eq. (6) in eq. (9) and solving for $N_{k}$ we have (see Appendix for the proof):

$$
N_{k}=\left[(1-\beta)+\frac{G_{k}}{\left(\frac{E_{b}}{N_{0}}\right)_{k}}\right] \frac{\left[\eta_{U L}(\delta+1)-\delta\right]}{\left[1-\beta\left(\eta_{U L}(\delta+1)-\delta\right)\right]}
$$

where $\delta=\frac{P_{\text {other }}}{P_{\text {noise }}}$.

If $\beta=0$ (interference cancellation is not applied) and $\delta=0$ (which means that $P_{\text {other }}=0$ ), then eq. (10) takes the form:

${ }^{3}$ If we assume the existence of perfect power control and the same $\left(\frac{E_{b}}{N_{0}}\right)$, data rate $R$, activity factor $v$ and consequently processing gain $G$ and total received power $p$ for all service-classes then eq. (6) takes the form $P_{\text {own }}=\frac{N\left(P_{\text {other }}+P_{\text {noise }}\right)}{(1-\beta)-N(1-\beta)+\frac{G}{\left(\frac{E_{b}}{N_{0}}\right)}}$ where $N$ is the total number of users in the reference cell. 
$N_{k}=\left[1+\frac{G_{k}}{\left(\frac{E_{b}}{N_{0}}\right)_{k}}\right] \eta_{U L}$

which is eq. (3) of [11] and shows the maximum number of service-class $k$ calls in an isolated cell.

\section{Note 2:}

If we assume the existence of perfect power control and the same $\left(\frac{E_{b}}{N_{0}}\right)$, data rate $R$, activity factor $v$ and consequently processing gain $G$ and total received power $p$ for all service-classes then eq. (10) takes the form:

$$
N=\left[(1-\beta)+\frac{G}{\left(\frac{E_{b}}{N_{0}}\right)}\right] \frac{\left[\eta_{U L}(\delta+1)-\delta\right]}{\left[1-\beta\left(\eta_{U L}(\delta+1)-\delta\right)\right]}
$$

where $\delta=\frac{P_{\text {other }}}{P_{\text {noise }}}$ and $N$ is the total number of users in the reference cell.

Now:

1) if $\beta=0$ then eq. (12) becomes:

$$
N=\left[1+\frac{G}{\left(\frac{E_{b}}{N_{0}}\right)}\right]\left[\eta_{U L}(\delta+1)-\delta\right]
$$

Equation (13) is exactly the same with eq. (8.12) of [2], if we prove that:

$$
\eta_{U L}(\delta+1)-\delta=\frac{n_{U L}}{i+1}
$$

where $i=\frac{P_{\text {other }}}{P_{\text {own }}}$.

The proof requires some calculations but it is trivial and thus is omitted.

2) if $\beta>0$, eq. (12) gives the same results with eq. (8) of [21]. To prove it, we should show that the ratio $\frac{\left[\eta_{U L}(\delta+1)-\delta\right]}{\left[1-\beta\left(\eta_{U L}(\delta+1)-\delta\right)\right]}$ of eq. (12) is the same with the ratio $\frac{\eta_{U L}}{1+i-\beta \eta_{U L}}$ of eq. (8) of [21] where $i=\frac{P_{\text {other }}}{P_{\text {own }}}$. Based on eq. (14), it is easy to show that these ratios are equal.

2.3. Determination of the uplink capacity and the bandwidth requirement of serviceclass $k$ calls

Having determined $N_{k}$ according to the proposed eq. (10), we continue by calculating the spread data rate $R_{s, k}$ of service-class $k$, as the proportion of $W$ which is utilised by a call of service-class $k$ : 
$R_{s, k}=\frac{W}{N_{k}}$

Now, we transform the chip rate $W$ to the uplink capacity $C$, and the spread data rate $R_{s, k}$ to the corresponding bandwidth requirement per call, $b_{k}$, of each service-class $k$. To achieve this, we define a basic bandwidth unit $(b b u)$. This $b b u$ can be determined as the greatest common divisor of the required call resources of all service-classes, or it can take an arbitrarily chosen small value. As an example, if $b b u=20 \mathrm{Kcps}$ (arbitrarily chosen), then $C$ and $b_{k}$ are given by:

$C=\left\lceil\frac{W}{b b u}\right\rceil=192$ channels, $b_{k}=\left\lceil\frac{R_{s, k}}{b b u}\right\rceil$ channels

Consider now an example of $K=4$ service-classes accommodated in a W-CDMA cell. In Table I, we present the results of $N_{k}$ and $R_{s, k}$, for $\eta_{U L}=0.5$ and $i=0.55$, when the calculation of $N_{k}$ is based either on eq. (10) or eq. (11). Equation (11), which does not take into account the other-cell interference, overestimates the maximum number of service-class $k$ calls in the cell, compared to the results of the proposed eq. (10). On the other hand, the results of eq. (10) show that the increase of the interference cancellation efficiency $\beta$ (from 0.5 to 0.8 ), leads to the increase of $N_{k}$ as expected.

Table I: Maximum number of calls and corresponding spread data rate when $\eta_{U L}=0.5, i=0.55, \delta=0.5$ (when $\beta>0$ ) and various values of the IC efficiency $\beta$.

\begin{tabular}{|c|c|c|c|c|c|c|c|c|c|c|c|c|}
\hline \multirow[b]{2}{*}{$\begin{array}{l}\text { Service- } \\
\text { class } k\end{array}$} & \multirow[b]{2}{*}{$\begin{array}{c}R_{k} \\
\text { (kbps) }\end{array}$} & \multirow[b]{2}{*}{$v_{k}$} & \multirow[b]{2}{*}{$\begin{array}{l}\left(\frac{E_{b}}{N_{0}}\right)_{k} \\
\text { (in dB) }\end{array}$} & \multirow[b]{2}{*}{$\left(\frac{E_{b}}{N_{0}}\right)_{k}$} & \multicolumn{2}{|c|}{$\begin{array}{l}\beta=0, \delta=0 \\
{[10]-[11]}\end{array}$} & \multicolumn{2}{|c|}{$\begin{array}{c}\beta=0.2 \\
\text { (proposed) }\end{array}$} & \multicolumn{2}{|c|}{$\begin{array}{c}\beta=0.5 \\
\text { (proposed) }\end{array}$} & \multicolumn{2}{|c|}{$\begin{array}{c}\beta=0.8 \\
\text { (proposed) }\end{array}$} \\
\hline & & & & & $\begin{array}{c}N_{k} \\
\text { (eq. 11) }\end{array}$ & $\begin{array}{c}R_{s, k} \\
\text { (eq. 15) }\end{array}$ & $\begin{array}{c}N_{k} \\
\text { (eq. 10) }\end{array}$ & $\begin{array}{c}R_{s, k} \\
\text { (eq. 15) }\end{array}$ & $\begin{array}{c}N_{k} \\
\text { (eq. 10) }\end{array}$ & $\begin{array}{c}R_{s, k} \\
\text { (eq. 15) }\end{array}$ & $\begin{array}{c}N_{k} \\
\text { (eq. 10) }\end{array}$ & $\begin{array}{c}R_{s, k} \\
\text { (eq. 15) }\end{array}$ \\
\hline 1 & 7.95 & 0.67 & 4.0 & 2.51 & 144 & 26.67 & 75.7 & 50.70 & 82.1 & 46.75 & 89.7 & 42.78 \\
\hline 2 & 7.95 & 0.67 & 7.0 & 5.01 & 72.4 & 53.02 & 38.1 & 100.88 & 41.2 & 93.11 & 45.0 & 85.31 \\
\hline 3 & 32 & 1.0 & 3.0 & 2.00 & 30.6 & 125.61 & 16.0 & 239.44 & 17.3 & 221.63 & 18.9 & 203.64 \\
\hline 4 & 64 & 1.0 & 2.0 & 1.58 & 19.4 & 197.65 & 10.2 & 377.47 & 10.9 & 350.39 & 11.9 & 322.88 \\
\hline
\end{tabular}

\section{Call blocking probabilities assuming Poisson arrivals}

\subsection{A system with hard blocking only}

In connection-oriented systems, every system state $j(j=0,1, \ldots, C)$ can be a non-blocking or a blocking state for service-class $k$ calls, depending on the bandwidth requirement $b_{k}$. Assuming Poisson arriving calls, exponentially distributed service times and the CS bandwidth allocation policy, then the EMLM results. In the EMLM, the un-normalized values of the system state probabilities, $q(j)$, can be determined by the following accurate and recursive formula, known in the literature as Kaufman-Roberts formula (or recursion) [13], [14]:

$q(j)= \begin{cases}1 & , \text { for } j=0 \\ \frac{1}{j} \sum_{k=1}^{K} a_{k} b_{k} q\left(j-b_{k}\right), & \text { for } j=1, \ldots, C \\ 0 & , \text { otherwise }\end{cases}$ 
where $\alpha_{k}=\lambda_{k} / \mu_{k}$ is the offered traffic-load of service-class $k$ calls (in erl) while $\lambda_{k}$ and $\mu_{k}$ are the mean arrival and service rate of service-class $k$ calls, respectively.

The calculation of TC probabilities of service-class $k, P_{b_{k}}$, is based on the following formula:

$P_{b_{k}}=\sum_{j=C-b_{k}+1}^{C} G^{-1} q(j)$

where $G=\sum_{j=0}^{C} q(j)$ is the normalization constant.

Note that in the case of Poisson traffic, TC probabilities coincide with CC probabilities due to PASTA [8], [24].

\subsection{A system with both hard and soft blocking}

In CDMA networks we distinguish two types of states $j$ : a) those states that are certainly blocking states for service-class $k$ calls (hard blocking states) and b) those states that are blocking states for service-class $k$ calls with a probability $0<L_{j, k}<1$ (soft or local blocking states) due to the stochastic nature of the other-cell interference. In what follows, we show how we can incorporate the notion of local (or soft) blocking in eq. (17).

To consider in the reference cell the other-cell interference, we approximate it by an independent, lognormally distributed random variable with parameters $\mu$ and $\sigma$, given by:

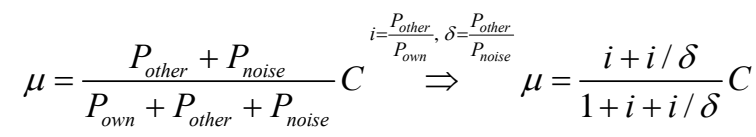

$\sigma=\mu$

Note that the parameter $\mu$ is chosen to be equal to $\sigma$ as proposed in the literature (e.g., [9][12], [25]-[26]). The value of $\mu$ expresses the average capacity that is lost from the reference cell due to the other-cell interference and noise.

If the thermal noise $P_{\text {noise }}$ is not considered, then eq. (19) takes the form (see also eq. (27) in [11]):

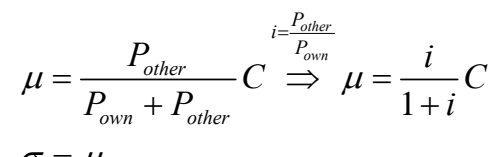

$\sigma=\mu$

The local blocking probability in state $j$, denoted as $L_{j}$, expresses the probability that the other-cell interference is greater than the available capacity $(C-j)$ of the reference cell and is independent of the bandwidth requirement of service-class $k$ calls [9]:

$$
\begin{aligned}
& L_{j}=P\left(j^{\prime}>C-j\right)=1-P\left(j^{\prime}<C-j\right) \Rightarrow \\
& L_{j}=1-C D F(C-j)
\end{aligned}
$$

where $j^{\prime}$ denotes the occupied channels due to the other cell interference and $C D F(x)$ is the cumulative distribution function of the lognormal distribution.

The values of $C D F(x)$ can be determined by: 
$C D F(x)=\frac{1}{2}\left(1+e r f\left(\frac{\ln (x)-M}{S \sqrt{2}}\right)\right)$

where erf is the error function, while $M$ and $S$ refer to the parameters of the normal distribution and are given by:

$$
\begin{aligned}
& M=\ln \left(\frac{\mu^{2}}{\sqrt{\mu^{2}+\sigma^{2}}}\right) \\
& S=\sqrt{\ln \left(1+\frac{\sigma^{2}}{\mu^{2}}\right)}
\end{aligned}
$$

Consider now a new service-class $k$ call which requires $b_{k}$ channels in order to be accepted in the cell. We can express the passage factor $1-L_{j, b_{k}}$, i.e., the probability that the call will not be blocked due to the other-cell interference, as a function of the number of channels occupied in the cell and the bandwidth requirement $b_{k}$ [9], [11]:

$$
1-L_{j, b_{k}}=\prod_{x=j}^{j+b_{k}-1}\left(1-L_{x}\right)=\left(1-L_{j}\right)\left(1-L_{j+1}\right)\left(1-L_{j+2}\right) \ldots\left(1-L_{j+b_{k}-1}\right)
$$

Note that the right hand side of eq. (25) consists of $b_{k}$ terms of the form $\left(1-L_{x}\right)$. This means that every time a service-class $k$ call obtains one channel, the value of the local blocking probability changes (e.g., if in state $x$, the value of $1-L_{x}$ becomes 1- $L_{x+1}$ etc.) and the call is finally accepted in the cell if all $b_{k}$ channels are assigned to the call.

In [10], a different and less complicated approach is proposed. A new service-class $k$ call will be accepted in the cell if all $b_{k}$ channels are assigned to the call simultaneously. This means, that the other-cell interference (and consequently the local blocking probability) remains the same during the allocation process of these $b_{k}$ channels. In that case, the passage factor $1-L_{j, b_{k}}$ is equal to the last term of eq. (25), i.e.,:

$1-L_{j, b_{k}}=\left(1-L_{j+b_{k}-1}\right)$

Herein we adopt the approach of [10] as it is more realistic for the call admission control of CDMA systems.

Due to the introduction of the passage factor according to eq. (26), the transition rate from state $\left(j-b_{k}\right)$ to state $j$, equals $\left(1-L_{j-b_{k}, b_{k}}\right) \lambda_{k}=\left(1-L_{j-1}\right) \lambda_{k}$. Figure 1 presents the system's state transition diagram which is depicted by a one-dimensional Markov chain. Note that $y_{k}(j)$ denotes the average number of service-class $k$ calls in state $j$.

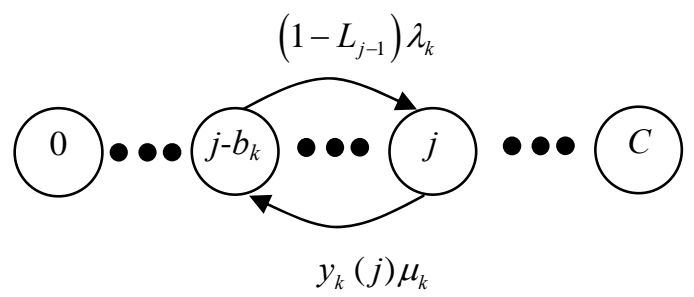

Figure 1: State transition diagram of Poisson service-class $k$ calls with local blocking between states $j-b_{k}$ and $j$. 
The un-normalized values of the system state probabilities, $q(j)$, are given by the following approximate but recursive formula [10]:

$q(j)= \begin{cases}1 & , \text { for } j=0 \\ \frac{1}{j} \sum_{k=1}^{K} a_{k} b_{k} q\left(j-b_{k}\right)\left(1-L_{j-b_{k}, b_{k}}\right), & \text { for } j=1, \ldots, C \\ 0 \quad, & \text { otherwise }\end{cases}$

where: $\alpha_{k}=\lambda_{k} / \mu_{k}$ is the offered traffic-load of service-class $k$ calls (in erl) and the values of $\left(1-L_{j-b_{k}, b_{k}}\right)$ are given by eq. (26).

The determination of TC probabilities of service-class $k, P_{b_{k}}$, is based on the formula [10]:

$P_{b_{k}}=\sum_{j=0}^{C} G^{-1} L_{j, j+b_{k}} q(j)$

where $G=\sum_{j=0}^{C} q(j)$ is the normalization constant.

To summarize the differences between [9] (or [11]), [10] and the proposed method, we present in Table II the algorithmic steps for the calculation of TC probabilities in each case.

Table II: Algorithms for the calculation of TC probabilities in the case of Poisson traffic.

\begin{tabular}{|l||l||l|}
\hline $\begin{array}{l}\text { Algorithm for the calculation of TC } \\
\text { probabilities based on [9], [11] }\end{array}$ & $\begin{array}{l}\text { Algorithm for the calculation of TC } \\
\text { probabilities based on [10] }\end{array}$ & $\begin{array}{l}\text { Proposed algorithm for the calculation } \\
\text { of TC probabilities }\end{array}$ \\
\hline \hline 1) Determine $N_{k}$ (eq. (11)) & 1) Determine $N_{k}$ (eq. (11)) & 1) Determine $N_{k}$ (eq. (10)) \\
\hline 2) Determine $R_{s, k}$ (eq. (15)) & 2) Determine $R_{s, k}$ (eq. (15)) & 2) Determine $R_{s, k}$ (eq. (15)) \\
\hline 3) Determine $C$, $b_{k}$ (eq. (16)) & 3) Determine $C, b_{k}$ (eq. (16)) & 3) Determine $C, b_{k}$ (eq. (16)) \\
\hline 4) Determine $\mu, \sigma$ (eq. (20)) & 4) Determine $\mu, \sigma$ (eq. (20)) & 4) Determine $\mu, \sigma$ (eq. (19)) \\
\hline 5) Determine $L_{j}$ (eqs. (21-24)) & 5) Determine $L_{j}$ (eqs. (21-24)) & 5) Determine $L_{j}$ (eqs. (21-24)) \\
\hline 6) Determine $1-L_{j, b_{k}}$ (eq. (25)) & 6) Determine $1-L_{j, b_{k}}$ (eq. (26)) & 6) Determine $1-L_{j, b_{k}}$ (eq. (26)) \\
\hline 7) Determine $q(j)($ eq. (27)) & 7) Determine $q(j)($ eq. (27)) & 7) Determine $q(j)($ eq. (27)) \\
\hline 8) Determine $P_{b_{k}}$ (eq. 28)) & 8) Determine $P_{b_{k}}$ (eq. 28) & 8) Determine $P_{b_{k}}($ eq. 28)) \\
\hline
\end{tabular}

Consider again the example of the four service-classes presented in section 2.3. All calls arrive in the cell according to a Poisson process and have an exponentially distributed service time. The offered traffic-loads of all service classes are: $\alpha_{1}=8.0 \mathrm{erl}, \alpha_{2}=4.0 \mathrm{erl}, \alpha_{3}=0.5 \mathrm{erl}$ and $\alpha_{4}=1.0$ erl. Assuming now that the $b b u=13.5$ Kcps we obtain the values of $C$ and $b_{k}$ $(k=1, \ldots, 4)$ :

a) Based on the $7^{\text {th }}$ column of Table I ( $\beta=\delta=0$ ): $C=284, b_{1}=2, b_{2}=4, b_{3}=10$ and $b_{4}=15$ channels. These values will be used for the calculation of TC probabilities in the case of [9] (or [11]) and [10].

b) Based on the $9^{\text {th }}$ column of Table I ( $\left.\beta=0.2, \delta=0.5\right)$ : $C=284, b_{1}=4, b_{2}=8, b_{3}=18$ and $b_{4}=28$ channels.

c) Based on the $11^{\text {th }}$ column of Table I $(\beta=0.5, \delta=0.5)$ : $C=284, b_{1}=4, b_{2}=7, b_{3}=17$ and $b_{4}=26$ channels. 
d) Based on the $13^{\text {th }}$ column of Table I $(\beta=0.8, \delta=0.5)$ : $C=284, b_{1}=4, b_{2}=7, b_{3}=16$ and $b_{4}=24$ channels.

The values of (b), (c) and (d) will be used for the calculation of TC probabilities in the case of the proposed algorithm.

In the x-axis of Figs. 2-7, $\alpha_{1}, \alpha_{2}, \alpha_{3}$ and $\alpha_{4}$ increase in steps of 2.0, 1.0, 0.5 and 0.25 erl, respectively. So, in Point 1 we have: $\left(\alpha_{1}, \alpha_{2}, \alpha_{3}, \alpha_{4}\right)=(8.0,4.0,0.5,1.0)$, while in Point 13 $\left(\alpha_{1}, \alpha_{2}, \alpha_{3}, \alpha_{4}\right)=(32.0,16.0,6.5,4.0)$. In Figs. 2-3, we present the TC probabilities of all service-classes following [9] (or [11]) and [10], respectively. The results show that the approach of [9] (whereby a service-class $k$ call obtains one channel at a time up to $b_{k}$ channels) gives much higher TC probabilities compared to the more realistic approach of [10] (a service-class $k$ call obtains all $b_{k}$ channels simultaneously). In Figs. 4-7, we adopt the approach of [10] for the calculation of the passage factors, consider three values of the interference cancellation efficiency $\beta=0.2,0.5,0.8$ and show the TC probabilities for each service-class, respectively. The TC probabilities obtained by the proposed algorithm cannot be approximated by [9], [10] or [11], since a different approach is followed for the calculation of $N_{k}$ 's, $\mu$ and $\sigma$. In addition, the increase of the interference cancellation efficiency results in the decrease of TC probabilities as expected.

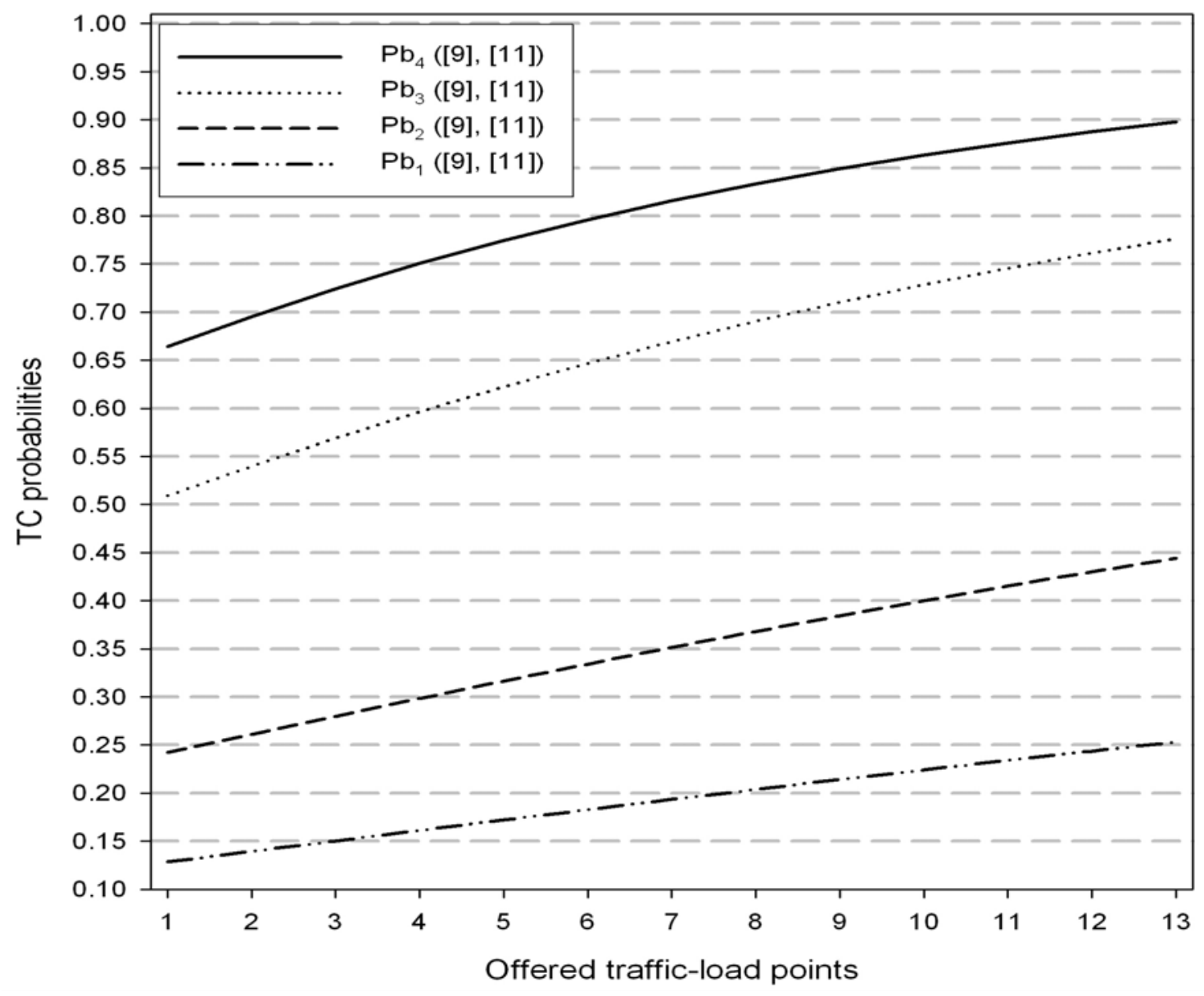

Figure 2: TC probabilities of all service-classes according to [9]-[11] (interference cancellation is not included). 


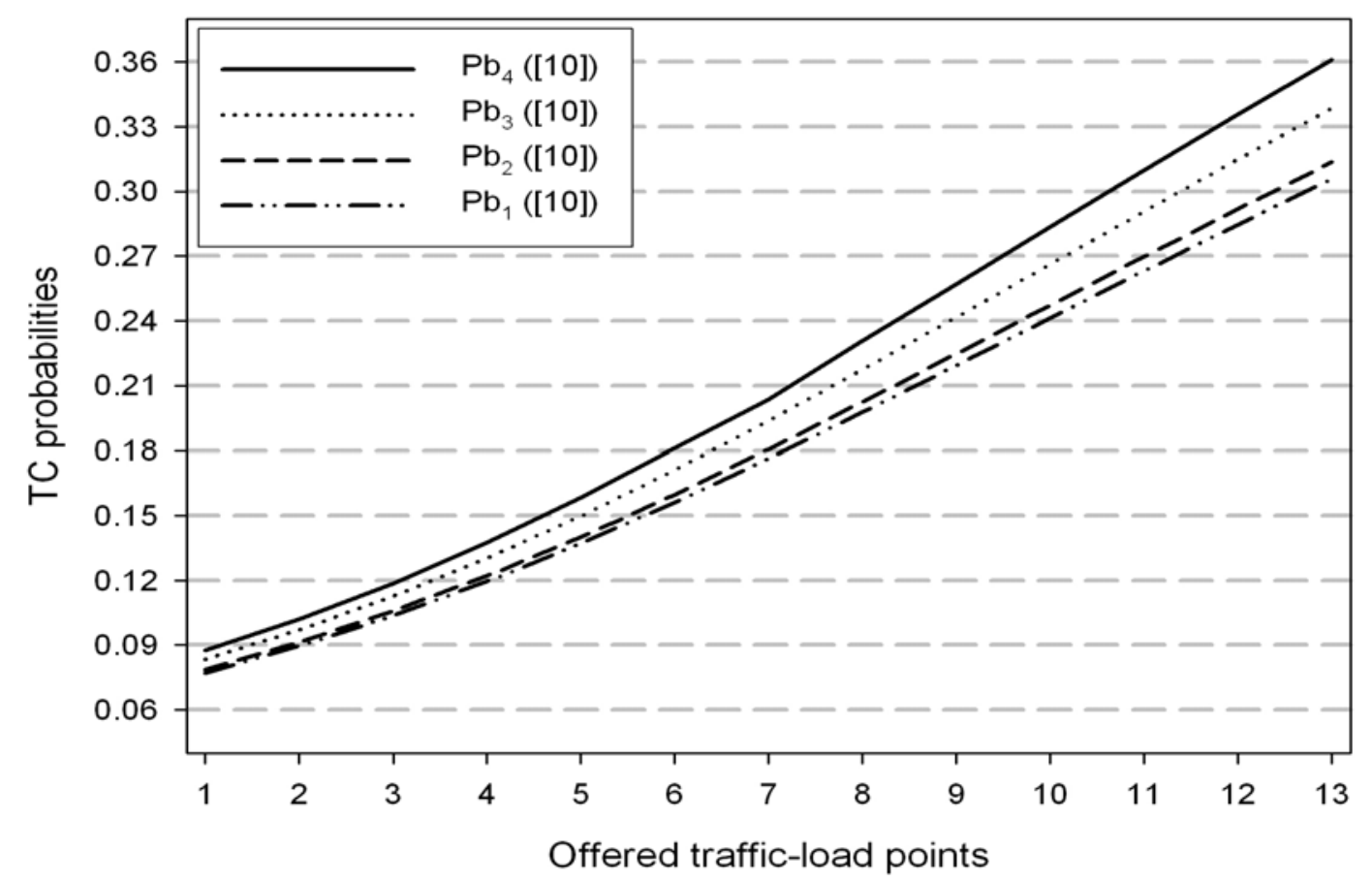

Figure 3: TC probabilities of all service-classes according to [10] (interference cancellation is not included).

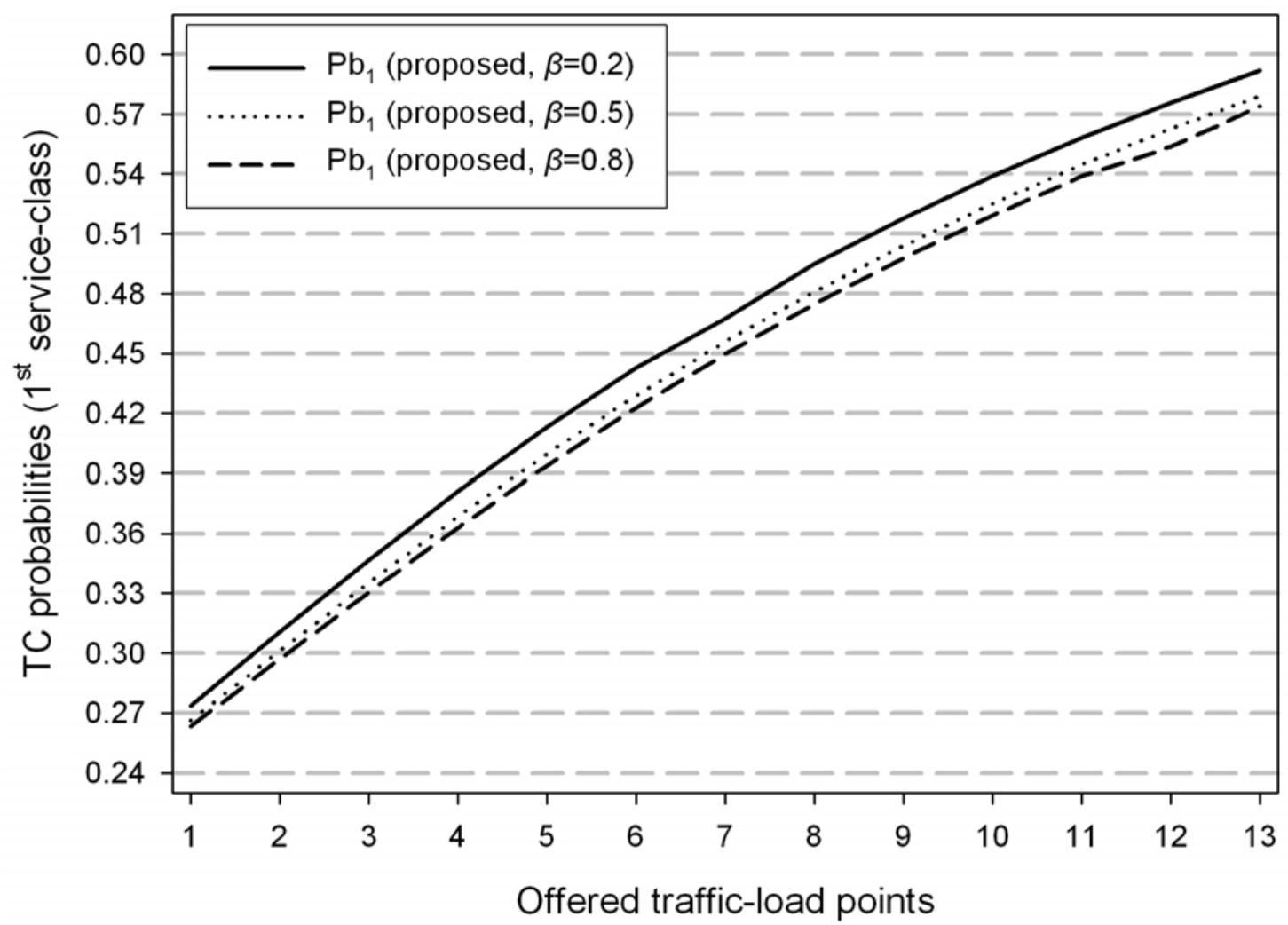

Figure 4: TC probabilities of the $1^{\text {st }}$ service-class for three different values of the interference cancellation efficiency. 


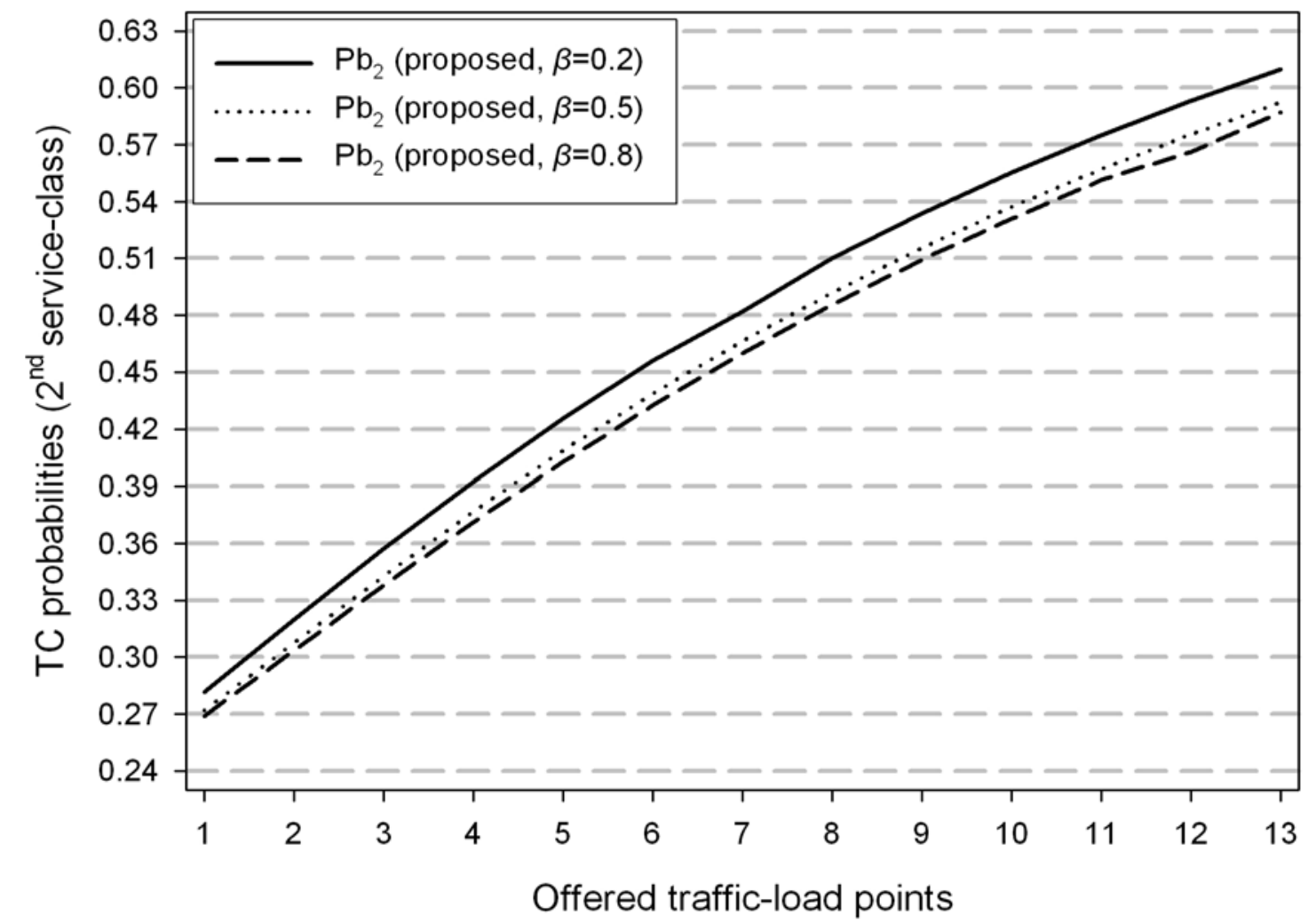

Figure 5: TC probabilities of the $2^{\text {nd }}$ service-class for three different values of the interference cancellation efficiency.

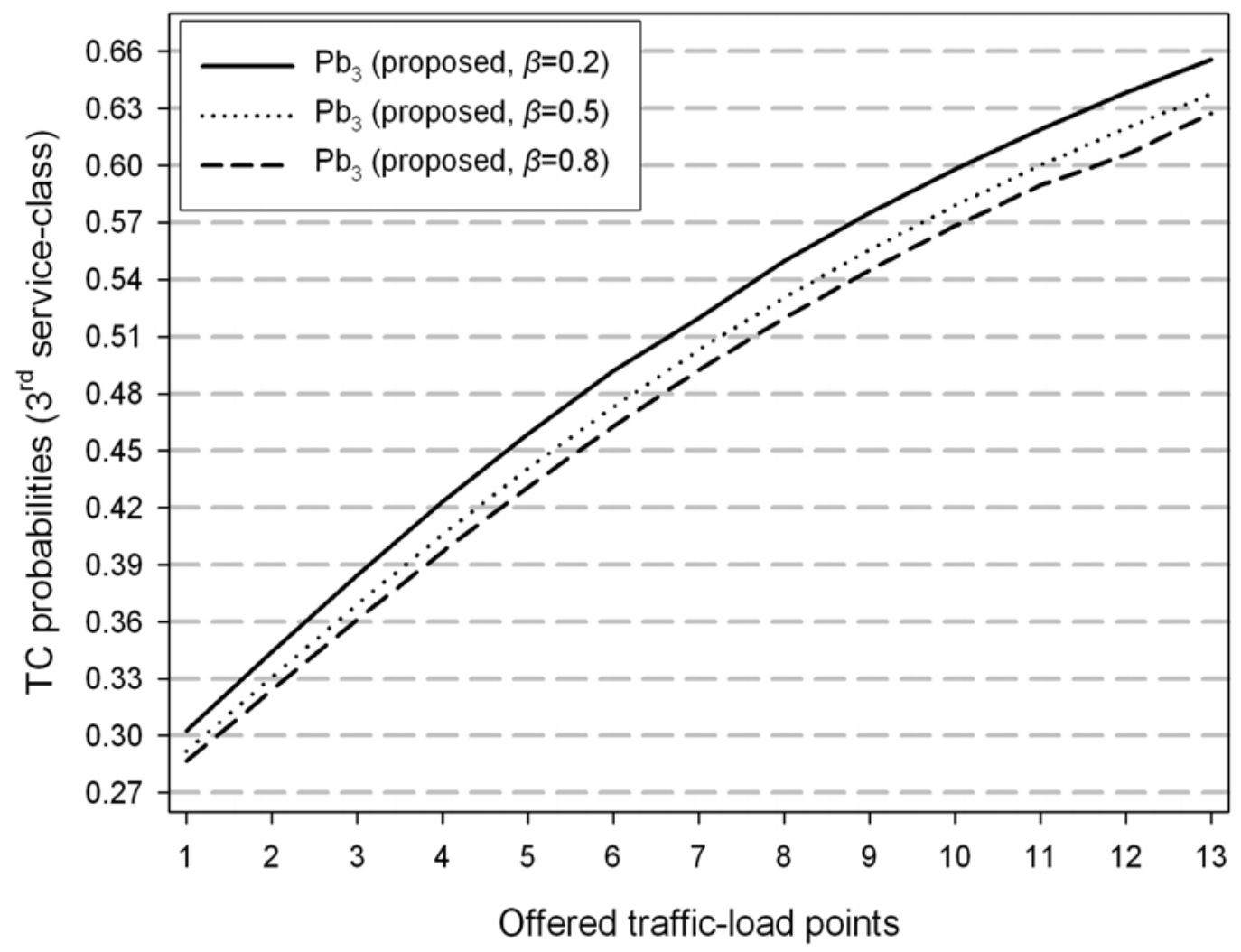

Figure 6: TC probabilities of the $3^{\text {rd }}$ service-class for three different values of the interference cancellation efficiency. 


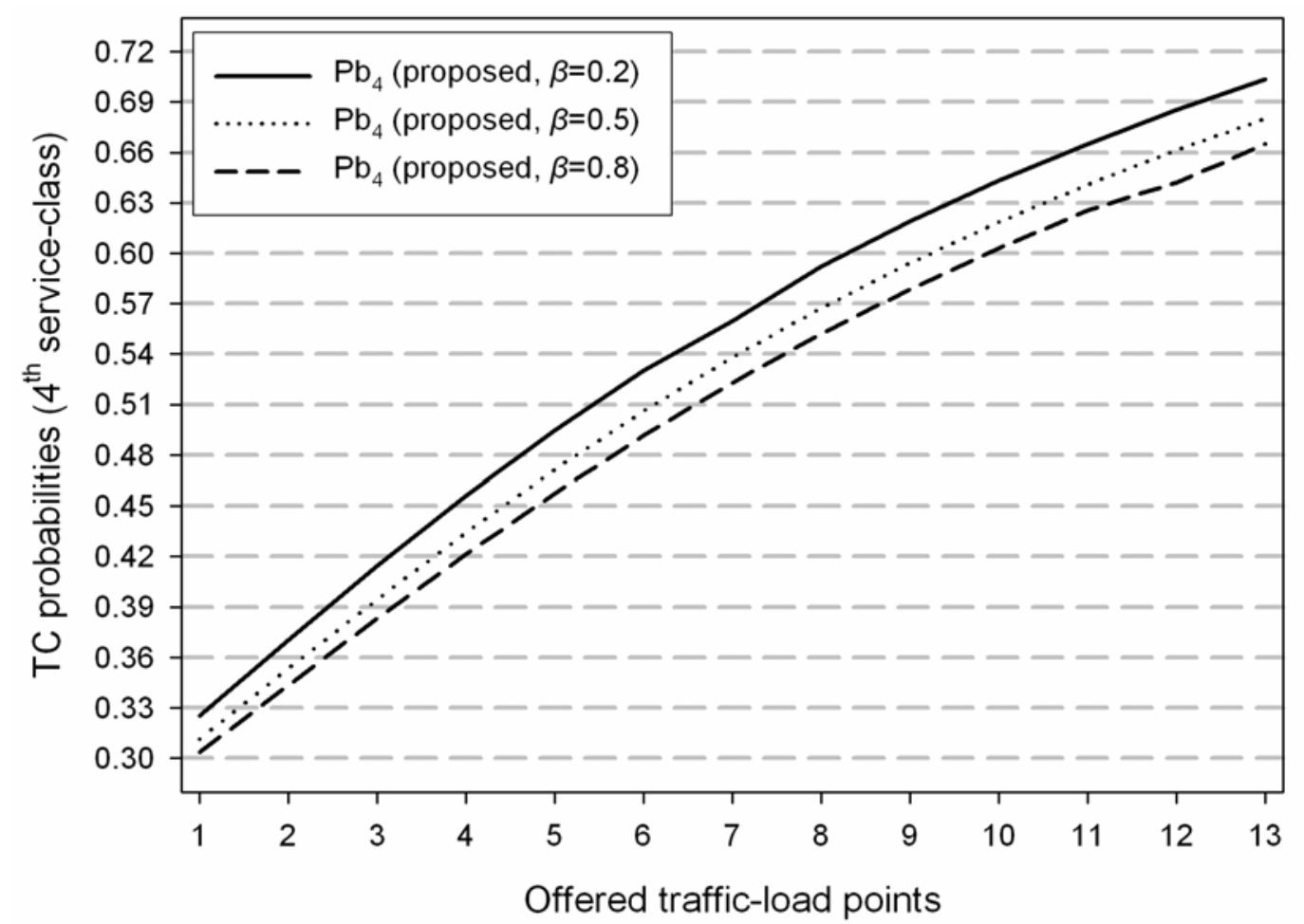

Figure 7: TC probabilities of the $4^{\text {th }}$ service-class for three different values of the interference cancellation efficiency.

\section{Congestion probabilities assuming batched Poisson arrivals}

\subsection{A system with hard blocking only}

Consider a system of capacity $C$ channels that accommodates calls of $K$ different serviceclasses. Calls of each service-class $k(k=1, \ldots, K)$ arrive in the system according to a batched Poisson process with arrival rate $\lambda_{k}$ and batch size distribution $B_{m}^{(k)}$, where $B_{m}^{(k)}$ denotes the probability that an arriving batch contains $m$ calls of service-class $k$. Each service-class $k$ call has a bandwidth requirement of $b_{k}$ channels and an exponentially distributed service time with mean $\mu_{k}^{-1}$. Assuming the partial batch blocking discipline for the call admission control the BP-EMLM results [17]. Based on [17], the un-normalized values of the system state probabilities, $q(j)$, are determined by the following accurate and recursive formula:

$$
q(j)=\left\{\begin{array}{lc}
1 & \text { for } j=0 \\
\frac{1}{j} \sum_{k=1}^{K} \alpha_{k} b_{k} \sum_{l=1}^{\left\lfloor j / b_{k}\right\rfloor} \hat{B}_{l-1}^{(k)} q\left(j-l b_{k}\right) & \text { for } j=1, \ldots, C \\
0 & \text { otherwise }
\end{array}\right.
$$

where: $\alpha_{k}=\lambda_{k} / \mu_{k}$ (in erl), $\left\lfloor j / b_{k}\right\rfloor$ is the largest integer less than or equal to $j / b_{k}$ and $\hat{B}_{l}^{(k)}$ is the complementary batch size distribution given by $\hat{B}_{l}^{(k)}=\sum_{m=l+1}^{\infty} B_{m}^{(k)}$.

Note that if $B_{m}^{(k)}=1$ for $m=1$ and $B_{m}^{(k)}=0$ for $m>1$ the arrival process is Poisson and the EMLM results. In that case, $q(j)$ 's are given by the Kaufman-Roberts recursion (eq. 17).

A highly used batch size distribution is the geometric distribution which is memoryless and a district equivalent of the exponential distribution. In that case, if service-class $k$ calls 
arrive in batches of size $s_{k}$ where $s_{k}$ is determined by the geometric distribution with parameter $\gamma_{k}$, i.e., $\operatorname{Pr}\left(s_{k}=r\right)=\left(1-\gamma_{k}\right) \gamma_{k}^{r-1}, r \geq 1$, then the model of [17] coincides with the model proposed by Delbrouck in [15], as shown in both [16], [17]. More precisely, since $\hat{B}_{l-1}^{(k)}=\gamma_{k}^{l-1}$, eq. (29) becomes:

$$
q(j)= \begin{cases}1 & \text { for } j=0 \\ \frac{1}{j} \sum_{k=1}^{K} \alpha_{k} b_{k} \sum_{l=1}^{\left\lfloor j / b_{k}\right\rfloor} \gamma_{k}^{l-1} q\left(j-l b_{k}\right) & \text { for } j=1, \ldots, C \\ 0 & \text { otherwise }\end{cases}
$$

Based on eq. (29) we can calculate:

a) The average number of service-class $k$ calls when the system state is $j, y_{k}(j)$ :

$$
y_{k}(j)=\alpha_{k} \sum_{l=1}^{\left\lfloor j / b_{k}\right\rfloor} \hat{B}_{l-1}^{(k)} q\left(j-l b_{k}\right) / q(j)
$$

b) The average number of service-class $k$ calls in the system, $\bar{n}_{k}$ :

$$
\bar{n}_{k}=\sum_{j=1}^{C} G^{-1} y_{k}(j) q(j)
$$

c) The CC probabilities of service-class $k$ calls, $C_{b_{k}}$ :

$$
C_{b_{k}}=\left(\alpha_{k} \hat{B}_{k}-\bar{n}_{k}\right) / \alpha_{k} \hat{B}_{k}
$$

where: $\hat{B}_{k}$ is the average batch size of service-class $k$ batches and is determined by: $\hat{B}_{k}=$ $\sum_{m=1}^{\infty} m B_{m}^{(k)}$.

Note that in the case of the geometric batch size distribution with parameter $\gamma_{k}$ we have: $\hat{B}_{k}=$ $\frac{1}{1-\gamma_{k}}$.

d) The TC probabilities of service-class $k$ calls, $P_{b_{k}}$, according to eq. (18).

\subsection{A system with both hard and soft blocking}

To introduce the notion of local blocking in the BP-EMLM of [17] we adopt the approach of [10]. Due to the batched Poisson process and the introduction of passage factors according to eq. (26), the transition rate from state $\left(j-l b_{k}\right)$ to state $j$, equals $\left(1-L_{j-b_{k}, b_{k}}\right) \lambda_{k} \hat{B}_{l-1}^{(k)}=\left(1-L_{j-1}\right) \lambda_{k} \hat{B}_{l-1}^{(k)}$, where $l=1,2, \ldots,\left\lfloor j / b_{k}\right\rfloor$. Figure 8 presents the corresponding system's state transition diagram, depicted as a one-dimensional Markov chain. Note that $y_{k}(j)$ denotes the average number of service-class $k$ calls in state $j$. 


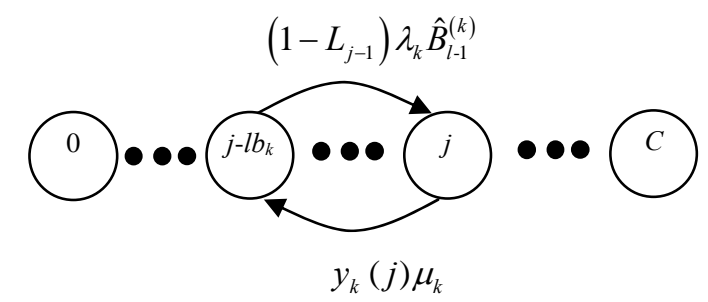

Figure 8: State transition diagram of batched Poisson service-class $k$ calls with local blocking between states $j$ - $l b_{k}$ and $j$.

As an example, if $j=4$ and $b_{k}=1$ then $l=1, \ldots, 4$ and the passage factors (towards state $j=4$ ) are the following: a) $\left(1-L_{3}\right) \lambda_{k} \hat{B}_{0}^{(k)}$ from state $j=3$ to $j=4$, b) $\left(1-L_{3}\right) \lambda_{k} \hat{B}_{1}^{(k)}$ from state $j=2$ to $j=4$, c) $\left(1-L_{3}\right) \lambda_{k} \hat{B}_{2}^{(k)}$ from state $j=1$ to $j=4$ and d) $\left(1-L_{3}\right) \lambda_{k} \hat{B}_{3}^{(k)}$ from state $j=0$ to $j=4$.

To calculate the un-normalized system state probabilities, $q(j)$, in a CDMA cell which accommodates batched Poisson calls of $K$ different service-classes under the partial batch blocking discipline, we propose the following approximate but recursive formula:

$$
q(j)= \begin{cases}1 & \text { for } j=0 \\ \frac{1}{j} \sum_{k=1}^{K} \alpha_{k} b_{k} \sum_{l=1}^{\left\lfloor j / b_{k}\right\rfloor} \hat{B}_{l-1}^{(k)} q\left(j-l b_{k}\right)\left(1-L_{j-b_{k}, b_{k}}\right) & \text { for } j=1, \ldots, C \\ 0 & \text { otherwise }\end{cases}
$$

where: $\alpha_{k}=\lambda_{k} / \mu_{k}$ (in erl), $\left\lfloor j / b_{k}\right\rfloor$ is the largest integer less than or equal to $j / b_{k}, \hat{B}_{l}^{(k)}$ is the complementary batch size distribution given by $\hat{B}_{l}^{(k)}=\sum_{m=l+1}^{\infty} B_{m}^{(k)}$ and $\left(1-L_{j-b_{k}, b_{k}}\right)=\left(1-L_{j-1}\right)$.

Note that if $B_{m}^{(k)}=1$ for $m=1$ and $B_{m}^{(k)}=0$ for $m>1$ then the arrival process is Poisson and $q(j)$ 's are given by eq. (27).

In the case of the geometric batch distribution with parameter $\gamma_{k}$, eq. (34) takes the form:

$$
q(j)= \begin{cases}1 & \text { for } j=0 \\ \frac{1}{j} \sum_{k=1}^{K} \alpha_{k} b_{k} \sum_{l=1}^{\left\lfloor j / b_{k}\right\rfloor} \gamma_{k}^{l-1} q\left(j-l b_{k}\right)\left(1-L_{j-b_{k}, b_{k}}\right) & \text { for } j=1, \ldots, C \\ 0 & \text { otherwise }\end{cases}
$$

Based on eq. (34), we can calculate:

a) The average number of service-class $k$ calls when the system state is $j, y_{k}(j)$ :

$$
y_{k}(j)=\alpha_{k} \sum_{l=1}^{\left\lfloor j / b_{k}\right\rfloor} \hat{B}_{l-1}^{(k)} q\left(j-l b_{k}\right)\left(1-L_{j-b_{k}, b_{k}}\right) / q(j)
$$

b) The average number of service-class $k$ calls in the system, $\bar{n}_{k}$, via eq. (32).

c) The CC probabilities of service-class $k$ calls, $C_{b_{k}}$, via eq. (33). Note that $C_{b_{k}}$ refers to the call congestion probability of an arbitrary call of a batch.

d) The TC probabilities of service-class $k$ calls, $P_{b_{k}}$, according to eq. (28). Note that $P_{b_{k}}$ coincides with the call congestion probability of the first call of a batch. 
To summarize the steps for the calculation of $q(j)$ 's in the case of the batched Poisson process, one can consider the $3^{\text {rd }}$ column of Table II. The only difference is on step 7 where $q(j)$ 's should be determined by eq. (34) (or eq. (35) if the geometric batch size distribution is required).

\section{Numerical results}

In this section, we compare the analytical results of TC and CC probabilities, obtained by the proposed model for the batched Poisson process, for various values of the interference cancellation efficiency. For comparison we also present the corresponding analytical results obtained in the case of the Poisson process. To improve the readability of figures of this section and since simulation and analytical results are very close, we only present analytical results in figure format. However, at the end of this section we present, for comparison, analytical and simulation CC probabilities results in table format. Simulations are carried via the SIMSCRIPT III language [27] and are mean values of 7 runs.

Consider a W-CDMA cell that accommodates calls of $K=3$ different service-classes. Calls arrive in the cell according to a batched Poisson process. The batch size distribution of all service-classes follows the geometric distribution with parameters: $\gamma_{1}=5 / 6, \gamma_{2}=0.750$ and $\gamma_{3}=0.600$, respectively. This means that the average batch size of the three service-classes is: $\hat{B}_{1}=6$ calls, $\hat{B}_{2}=4$ calls and $\hat{B}_{3}=2.5$ calls. As far as the service time of calls is concerned, it follows the exponential distribution with mean value $\mu_{1}^{-1}=\mu_{2}^{-1}=\mu_{3}^{-1}=1$. In Table III, we present the traffic characteristics of all service-classes. In addition, we assume that: $\eta_{U L}=0.75$, $i=0.35, \delta=2.0, b b u=13.5 \mathrm{kcps}$ while the interference cancellation efficiency $\beta$ takes the values 0.0, 0.3 and 0.7. In the x-axis of Figs $9-14$ and in the $1^{\text {st }}$ column of Tables IV-VI, the offered traffic load of the $1^{\text {st }}, 2^{\text {nd }}$ and $3^{\text {rd }}$ service-class increase in steps of 0.2, 0.1 and 0.05 erl, respectively. So, point 1 refers to: $\left(\alpha_{1}, \alpha_{2}, \alpha_{3}\right)=(0.2,0.1,0.05)$ while point 8 to: $\left(\alpha_{1}, \alpha_{2}, \alpha_{3}\right)=$ $(1.6,0.8,0.4)$

Table III: Traffic parameters of the three service-classes.

\begin{tabular}{|c|c|c|c|c|c|c|}
\hline & & & & & & \\
\hline $\begin{array}{c}\text { Service- } \\
\text { class } k\end{array}$ & $\begin{array}{c}R_{k} \\
(\mathrm{kbps})\end{array}$ & $v_{k}$ & $\begin{array}{c}\left(\frac{E_{b}}{N_{0}}\right)_{k} \\
(\mathrm{indB})\end{array}$ & $\left(\frac{E_{b}}{N_{0}}\right)_{k}$ & $\gamma_{k}$ & $\begin{array}{c}a_{k} \\
\text { (in erl) }\end{array}$ \\
\hline 1 & 7.95 & 0.67 & 4.0 & 2.51 & 0.833 & 0.2 \\
\hline 2 & 12.20 & 0.67 & 4.0 & 2.51 & 0.750 & 0.1 \\
\hline 3 & 32.00 & 1.0 & 3.0 & 2.00 & 0.600 & 0.05 \\
\hline
\end{tabular}

Figures 9-11 present the analytical TC probabilities of each service-class, while Figs. 1214 present the corresponding CC probabilities. Based on these results, we conclude that: 1) CC probabilities are always higher than TC probabilities in the case of the batched Poisson process. This result is expected and shows the consistency of the proposed model. 2) The 
increase of $\beta$ results in the decrease of TC and CC probabilities. This result is also expected, since the interference cancellation reduces the own-cell interference. 3) The TC and CC probabilities obtained by assuming Poisson arrivals fail to approximate the corresponding TC and CC probabilities in the case of the batched Poisson process. This result shows the necessity of the proposed model and is also expected, since a batched Poisson process is more "peaked" and "bursty" than a Poisson process.

In Tables IV-VI, we present the analytical and simulation CC probabilities results, for the last call of a service-class $k$ batch $(k=1,2,3)$ and for $\beta=0.0,0.3$ and 0.7 , respectively. The analytical calculation of this CC probability is given by the formula $C_{b_{k, l a s t}}=\left(1-\gamma_{k}\right) C_{b_{k}}$. Based on Tables IV-VI, we see that simulation results are quite satisfactory compared to analytical results. Of similar accuracy are the simulation results of Figs. 9-14 and thus not presented. Furthermore, we see that the increase of the interference cancellation efficiency $\beta$, decreases the CC probabilities of the last call of a service-class $k$ batch.

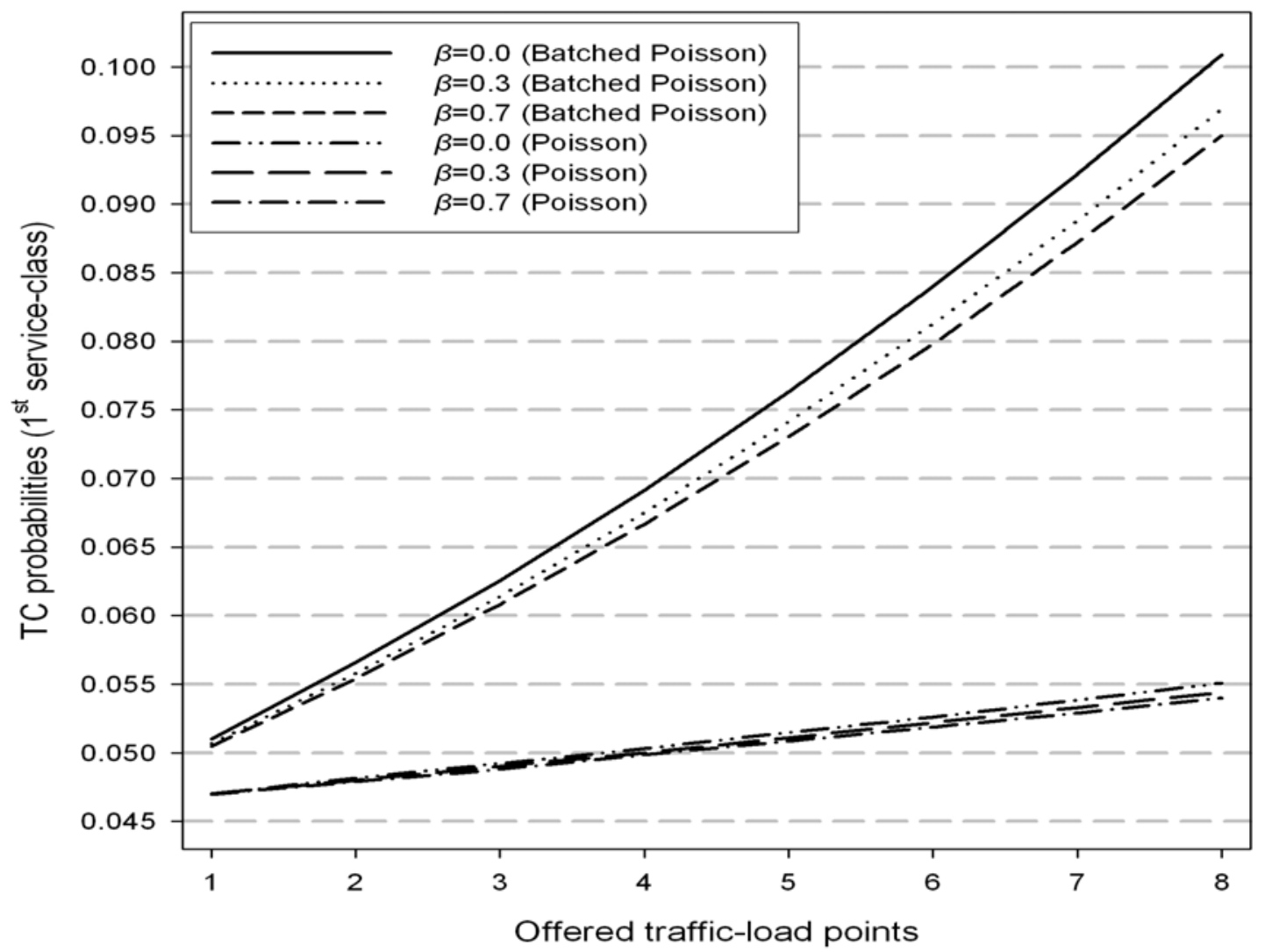

Figure 9: TC probabilities of the $1^{\text {st }}$ service-class. 


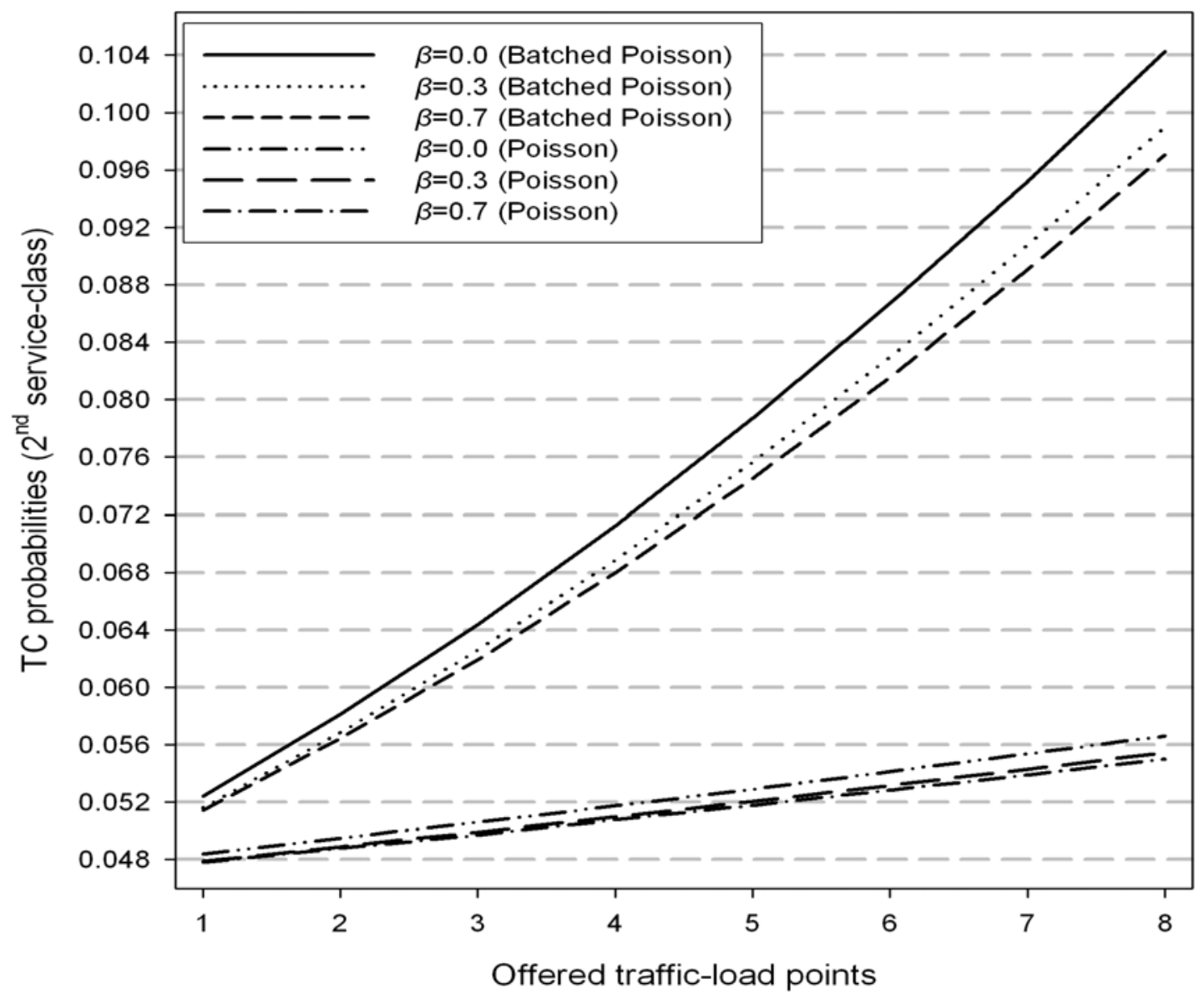

Figure 10: TC probabilities of the $2^{\text {nd }}$ service-class.

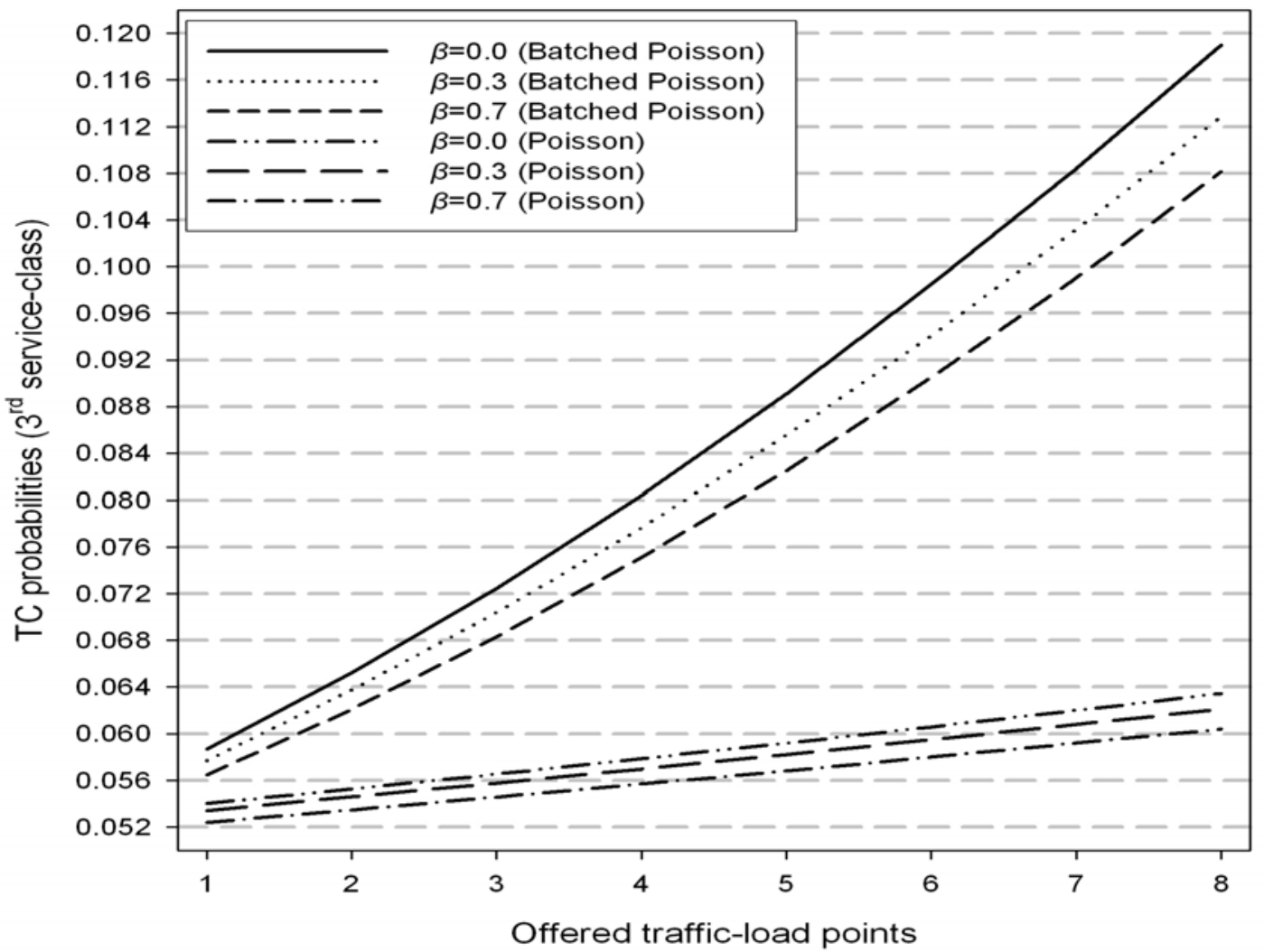

Figure 11: TC probabilities of the $3^{\text {rd }}$ service-class. 


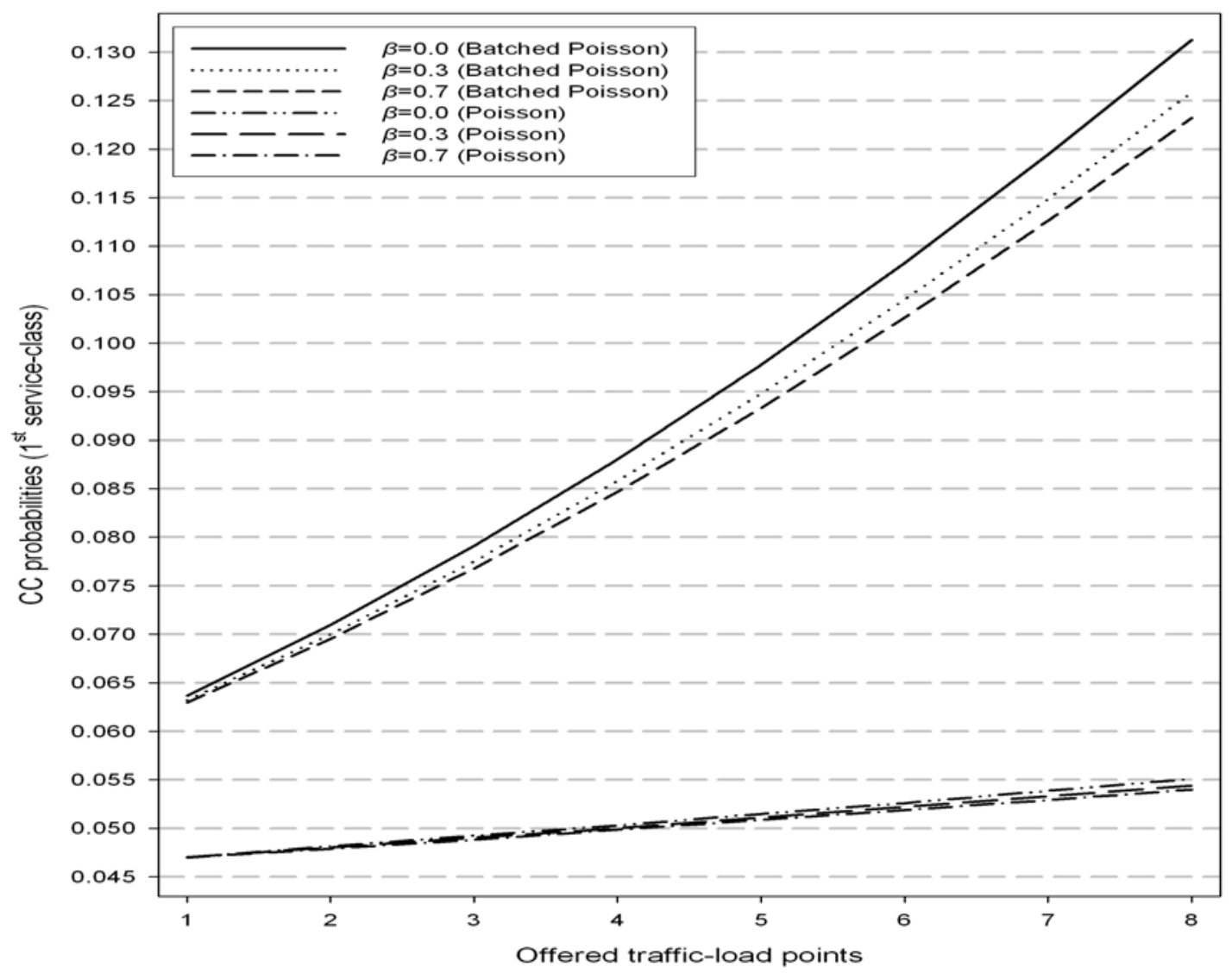

Figure 12: CC probabilities of the $1^{\text {st }}$ service-class.

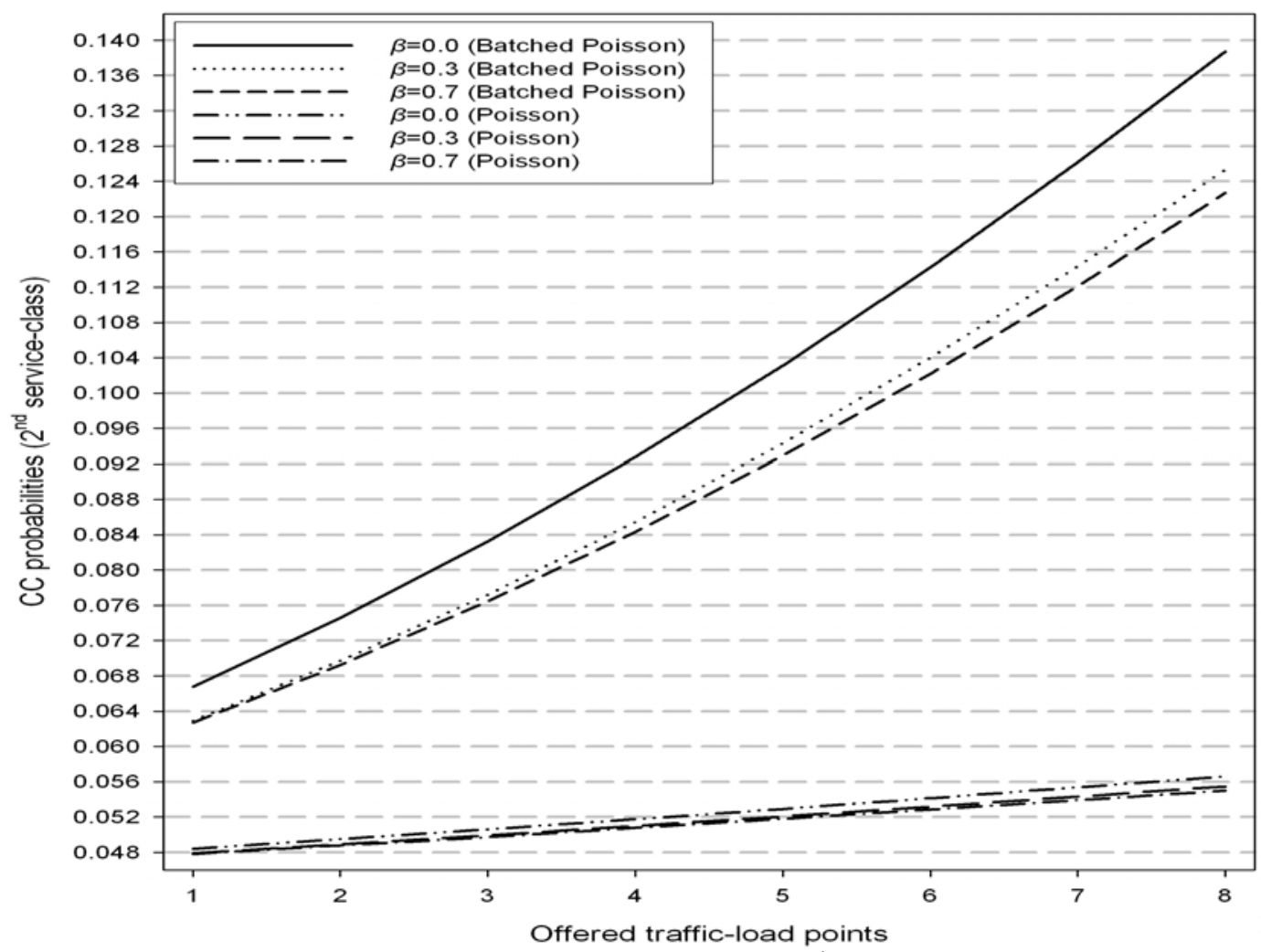

Figure 13: CC probabilities of the $2^{\text {nd }}$ service-class. 


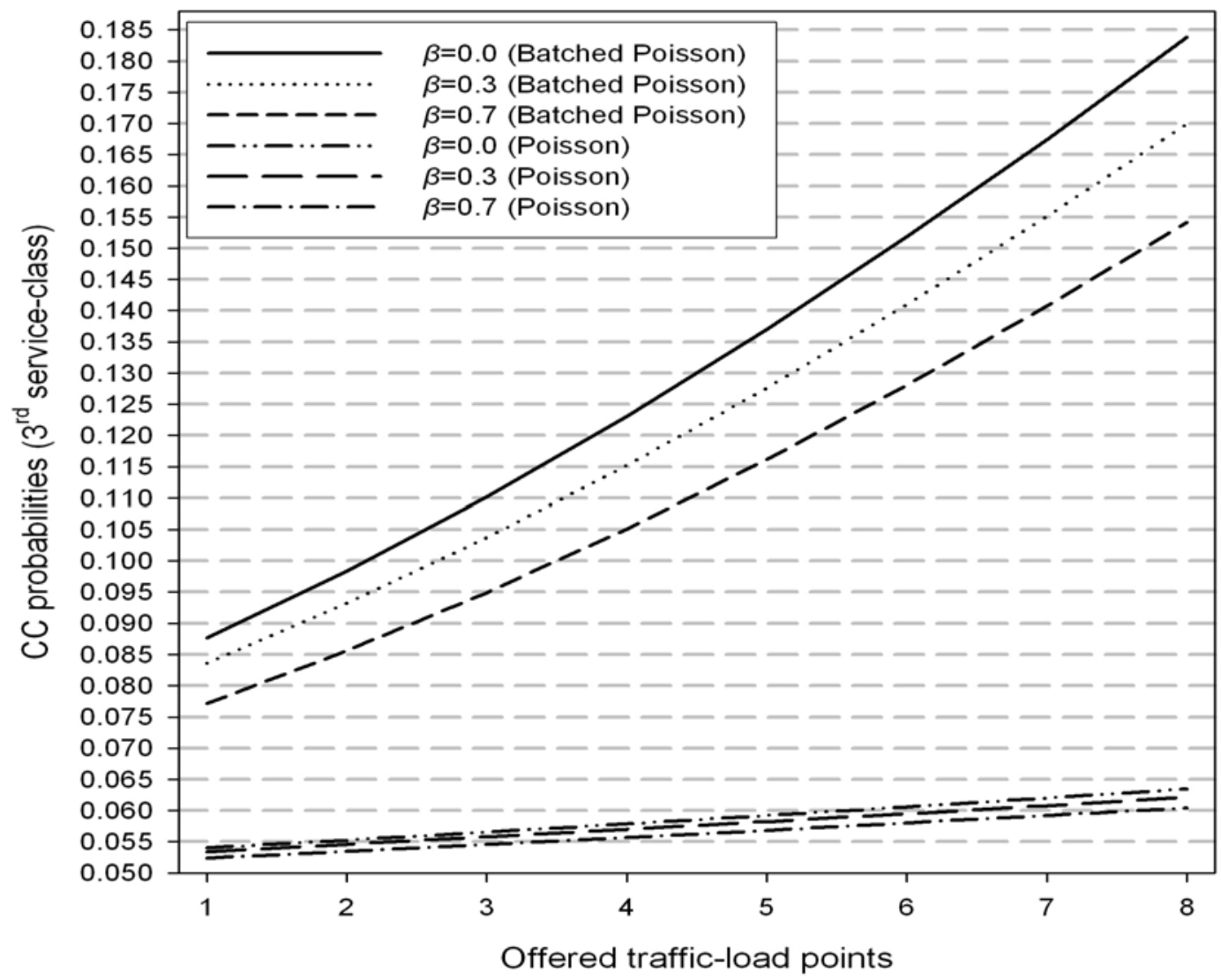

Figure 14: CC probabilities of the $3^{\text {rd }}$ service-class.

Table IV: CC probabilities of the last call of a batch (when $\beta=0.0$ )

\begin{tabular}{|c|c|c|c|c|c|c|}
\hline $\begin{array}{l}\text { Offered traffic- } \\
\text { load point }\end{array}$ & $\begin{array}{c}C_{b_{1}, \text { ast }} \\
\text { (analytical) }\end{array}$ & $\begin{array}{c}C_{b_{1} \text {,ast }} \\
\text { (simulation) }\end{array}$ & $\begin{array}{c}C_{b_{2, \text { last }}} \\
\text { (analytical) }\end{array}$ & $\begin{array}{c}C_{b_{2, \text { last }}} \\
\text { (simulation) }\end{array}$ & $\begin{array}{c}C_{b_{3, \text { last }}} \\
\text { (analytical) }\end{array}$ & $\begin{array}{c}C_{b_{3, \text { last }}} \\
\text { (simulation) }\end{array}$ \\
\hline 1 & 0.01061 & 0.01062 & 0.01669 & 0.01670 & 0.03506 & 0.03509 \\
\hline 2 & 0.01183 & 0.01185 & 0.01864 & 0.01866 & 0.03935 & 0.03939 \\
\hline 3 & 0.01318 & 0.01319 & 0.02081 & 0.02082 & 0.04408 & 0.04410 \\
\hline 4 & 0.01466 & 0.01468 & 0.02319 & 0.02320 & 0.04923 & 0.04927 \\
\hline 5 & 0.01629 & 0.01630 & 0.02577 & 0.02579 & 0.05479 & 0.05483 \\
\hline 6 & 0.01804 & 0.01805 & 0.02856 & 0.02857 & 0.06072 & 0.06077 \\
\hline 7 & 0.01990 & 0.01992 & 0.03153 & 0.03156 & 0.06698 & 0.06702 \\
\hline 8 & 0.02187 & 0.02188 & 0.03467 & 0.03469 & 0.07352 & 0.07356 \\
\hline
\end{tabular}

Table V: CC probabilities of the last call of a batch (when $\beta=0.3$ )

\begin{tabular}{|c|c|c|c|c|c|c|}
\hline $\begin{array}{l}\text { Offered traffic- } \\
\text { load point }\end{array}$ & $\begin{array}{c}C_{b_{1, \text { ast }}} \\
\text { (analytical) }\end{array}$ & $\begin{array}{c}C_{b_{1}, \text { last }} \\
\text { (simulation) }\end{array}$ & $\begin{array}{c}C_{b_{2, \text { last }}} \\
\text { (analytical) }\end{array}$ & $\begin{array}{c}C_{b_{2, \text { last }}} \\
\text { (simulation) }\end{array}$ & $\begin{array}{c}C_{b_{3, \text { last }}} \\
\text { (analytical) }\end{array}$ & $\begin{array}{c}C_{b_{3, \text { last }}} \\
\text { (simulation) }\end{array}$ \\
\hline 1 & 0.01053 & 0.01053 & 0.01573 & 0.01574 & 0.03344 & 0.03348 \\
\hline 2 & 0.01166 & 0.01167 & 0.01743 & 0.01745 & 0.03728 & 0.03731 \\
\hline 3 & 0.01292 & 0.01293 & 0.01930 & 0.01932 & 0.04148 & 0.04152 \\
\hline 4 & 0.01430 & 0.01431 & 0.02136 & 0.02137 & 0.04608 & 0.04612 \\
\hline 5 & 0.01580 & 0.01582 & 0.02357 & 0.02358 & 0.05104 & 0.05110 \\
\hline 6 & 0.01742 & 0.01743 & 0.02600 & 0.02603 & 0.05639 & 0.05643 \\
\hline 7 & 0.01914 & 0.01915 & 0.02859 & 0.02860 & 0.06204 & 0.06207 \\
\hline 8 & 0.02097 & 0.02099 & 0.03130 & 0.03134 & 0.06797 & 0.06801 \\
\hline
\end{tabular}


Table VI: CC probabilities of the last call of a batch (when $\beta=0.7$ )

\begin{tabular}{|c|c|c|c|c|c|c|}
\hline $\begin{array}{l}\text { Offered traffic- } \\
\text { load point }\end{array}$ & $\begin{array}{c}C_{b_{1, \text { last }}} \\
\text { (analytical) }\end{array}$ & $\begin{array}{c}C_{b_{1, \text { last }}} \\
\text { (simulation) }\end{array}$ & $\begin{array}{c}C_{b_{2, \text { last }}} \\
\text { (analytical) }\end{array}$ & $\begin{array}{c}C_{b_{2, \text { last }}} \\
\text { (simulation) }\end{array}$ & $\begin{array}{c}C_{b_{3, \text { last }}} \\
\text { (analytical) }\end{array}$ & $\begin{array}{c}C_{b_{3, \text { last }}} \\
\text { (simulation) }\end{array}$ \\
\hline 1 & 0.01049 & 0.01050 & 0.01568 & 0.01569 & 0.03086 & 0.03090 \\
\hline 2 & 0.01159 & 0.01159 & 0.01731 & 0.01732 & 0.03424 & 0.03429 \\
\hline 3 & 0.01279 & 0.01280 & 0.01910 & 0.01912 & 0.03795 & 0.03800 \\
\hline 4 & 0.01411 & 0.01411 & 0.02109 & 0.02110 & 0.04203 & 0.04206 \\
\hline 5 & 0.01555 & 0.01557 & 0.02322 & 0.02323 & 0.04646 & 0.04651 \\
\hline 6 & 0.01711 & 0.01712 & 0.02554 & 0.02556 & 0.05122 & 0.05126 \\
\hline 7 & 0.01877 & 0.01889 & 0.02802 & 0.02803 & 0.05630 & 0.05634 \\
\hline 8 & 0.02050 & 0.02052 & 0.03067 & 0.03069 & 0.06167 & 0.06170 \\
\hline
\end{tabular}

\section{Conclusion}

We propose a new multirate loss model for the call-level analysis of CDMA networks that support calls from different service-classes whose arrival follows a batched Poisson process. Calls are accepted in the system according to the partial batch blocking discipline. The new model takes into account the multiple access interference, the notion of local (soft) blocking, user's activity and the interference cancellation. Due to the existence of local blocking, the proposed model does not have a product form solution. However, we show an approximate but recursive formula for the calculation of occupancy distribution and consequently the determination of time and call congestion probabilities. In addition, we study the effect of the interference cancellation efficiency in these performance measures. Simulation results verify the accuracy of the proposed model.

\section{Appendix A}

\section{Proof of eq. (10)}

Substituting eq. (6) in eq. (9) we have: 


$$
\begin{aligned}
& \begin{array}{l}
\frac{N_{k}\left(P_{\text {other }}+P_{\text {noise }}\right)}{(1-\beta)-N_{k}(1-\beta)+\frac{G_{k}}{\left(\frac{E_{b}}{N_{0}}\right)_{k}}}+P_{\text {other }}+P_{\text {noise }} \\
\frac{P_{\text {noise }}}{1-\eta_{U L}} \Rightarrow
\end{array}
\end{aligned}
$$

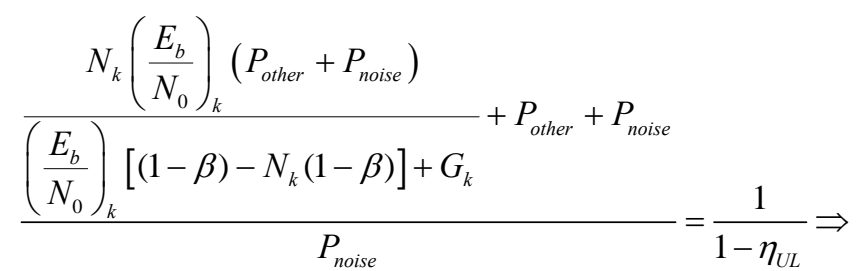

$$
\begin{aligned}
& \frac{\left(P_{\text {other }}+P_{\text {noise }}\right)\left[N_{k}\left(\frac{E_{b}}{N_{0}}\right)_{k}+\left(\frac{E_{b}}{N_{0}}\right)_{k}\left[(1-\beta)-N_{k}(1-\beta)\right]+G_{k}\right]}{P_{\text {noise }}\left[\left(\frac{E_{b}}{N_{0}}\right)_{k}\left[(1-\beta)-N_{k}(1-\beta)\right]+G_{k}\right]}=\frac{1}{1-\eta_{U L}} \stackrel{\substack{\delta=P_{\text {olver }} \\
\Rightarrow \text { mose }}}{\Rightarrow} \\
& (\delta+1)\left(1-\eta_{U L}\right)\left[N_{k}\left(\frac{E_{b}}{N_{0}}\right)_{k}+\left(\frac{E_{b}}{N_{0}}\right)_{k}\left[(1-\beta)-N_{k}(1-\beta)\right]+G_{k}\right]=\left[\left(\frac{E_{b}}{N_{0}}\right)_{k}\left[(1-\beta)-N_{k}(1-\beta)\right]+G_{k}\right] \Rightarrow
\end{aligned}
$$$$
N_{k}=\frac{\left(\frac{E_{b}}{N_{0}}\right)_{k}(1-\beta)+G_{k}-(\delta+1)\left(1-\eta_{U L}\right)\left[\left(\frac{E_{b}}{N_{0}}\right)_{k}(1-\beta)+G_{k}\right]}{\left(\frac{E_{b}}{N_{0}}\right)_{k}(\delta+1)\left(1-\eta_{U L}\right)[1-(1-\beta)]+\left(\frac{E_{b}}{N_{0}}\right)_{k}(1-\beta)} \Rightarrow
$$$$
N_{k}=\frac{\left[\left(\frac{E_{b}}{N_{0}}\right)_{k}(1-\beta)+G_{k}\right]}{\left(\frac{E_{b}}{N_{0}}\right)_{k}} \frac{\left[1-(\delta+1)\left(1-\eta_{U L}\right)\right]}{\left[\beta(\delta+1)\left(1-\eta_{U L}\right)+(1-\beta)\right]} \Rightarrow
$$$$
N_{k}=\frac{\left[\left(\frac{E_{b}}{N_{0}}\right)_{k}(1-\beta)+G_{k}\right]}{\left(\frac{E_{b}}{N_{0}}\right)_{k}} \frac{\left[\eta_{U L}(\delta+1)-\delta\right]}{\left[1+\beta\left(\delta-\eta_{U L} \delta-\eta_{U L}\right)\right]}
$$

or

$$
N_{k}=\left[(1-\beta)+\frac{G_{k}}{\left(\frac{E_{b}}{N_{0}}\right)_{k}}\right] \frac{\left[\eta_{U L}(\delta+1)-\delta\right]}{\left[1-\beta\left(\eta_{U L}(\delta+1)-\delta\right)\right]} \text { which is eq. (10). }
$$

\section{References}

[1] H.-H. Chen, The Next Generation CDMA Technologies, Wiley \& Sons, 2007.

[2] H. Holma and A. Toskala, eds., W-CDMA for UMTS - HSPA Evolution and LTE, $4^{\text {th }}$ edition, Wiley \& Sons, 2007.

[3] K. Fazel and S. Kaiser, Multi-Carrier and Spread Spectrum Systems: From OFDM and MC-CDMA to LTE and WiMAX, $2^{\text {nd }}$ edition, John Wiley \& Sons, 2008.

[4] D. B. Li, "The perspectives of large area synchronous CDMA technology for the fourth-generation mobile radio", IEEE Communications Magazine, vol. 41, no. 3, pp. 114-118, March 2003.

[5] U. Varshney, “4G Wireless Networks”, IT Professional, IEEE Computer Society, vol. 14, issue 5, pp. 34-39, September/October 2012. 
[6] S. Pinter and X. Fernando, "Estimation and equalization of fiber-wireless uplink for multiuser CDMA 4G networks”, IEEE Trans. Commun., vol. 58, no. 6, pp. 1803-1813, June 2010.

[7] S. Patel, C. Malhar, K. Kapadiya, “5G: Future Mobile Technology-Vision 2020”, International Journal of Computer Applications, vol. 54, no. 17, pp. 6-10, September 2012.

[8] K. Ross, Multiservice Loss Models for Broadband Telecommunication Networks, Springer, London, 1995.

[9] V. Iversen, V. Benetis, N. Ha, and S. Stepanov, "Evaluation of Multi-service CDMA Networks with Soft Blocking”, Proc. ITC Specialist Seminar, pp. 223-227, Antwerp, August/September 2004.

[10] L. Popova and W. Koch, "Analytical performance evaluation of mixed services with variable data rates for the uplink of UMTS”, Proc. ISWCS’06, Valencia, Spain, September 2006.

[11] V. Iversen, "Evaluation of Multi-service CDMA Networks with Soft Blocking”, Proc. of $3^{\text {rd }}$ Conference on Smart Spaces, ruSMART 2010, and $10^{\text {th }}$ Int. Conference, NEW2AN 2010, St. Petersburg, Russia, pp. 160-171, 23-25 August 2010.

[12] G. Kallos, V. Vassilakis and M. Logothetis, "Call-level performance analysis of a W-CDMA cell with finite population and interference cancellation”, European Transactions on Telecommunications, vol. 22, issue 1, pp. 25-30, January 2011.

[13] J. Kaufman, “Blocking in a shared resource environment”, IEEE Trans. Commun., vol. 29, no. 10, pp. 14741481, October 1981.

[14] J. Roberts, “A service system with heterogeneous user requirements”, in: G. Pujolle (Ed.), Performance of Data Communications systems and their applications, North Holland, Amsterdam, pp.423-431, 1981.

[15] L. Delbrouck, "On the steady state distribution in a service facility with different peakedness factors and capacity requirements”, IEEE Trans. Commun., 31 (11), pp. 1209-1211, November 1983.

[16] E. van Doorn and F. Panken, "Blocking Probabilities in a Loss System with Arrivals in Geometrically Distributed Batches and Heterogeneous Service Requirements”, IEEE/ACM Trans. Networking (1), pp. 664667, December 1993.

[17] J. Kaufman and K. Rege, "Blocking in a shared resource environment with batched Poisson arrival processes”, Performance Evaluation 24, pp. 249-263, February 1996.

[18] I. Moscholios and M. Logothetis, "The Erlang Multirate Loss Model with Batched Poisson Arrival Processes under the Bandwidth Reservation Policy”, Computer Communications, Vol. 33, Supplement 1, pp. S167S179, November 2010.

[19] I. Moscholios, J. Vardakas, M. Logothetis and A. Boucouvalas, "QoS Guarantee in a Batched Poisson Multirate Loss Model Supporting Elastic and Adaptive Traffic”, Proc. of IEEE ICC 2012, Ottawa, Canada, 10-15 June 2012.

[20] I. Moscholios, J. Vardakas, M. Logothetis and A. Boucouvalas, "Congestion Probabilities in a Batched Poisson Multirate Loss Model Supporting Elastic and Adaptive Traffic”, Annals of Telecommunications, Vol. 68, Issue 5, 2013, pp. 327-344.

[21] C. Rosa, T. Sorensen, J. Wigard and R. Mogensen, "Interference cancellation and 4-branch antenna diversity for W-CDMA uplink packet access”, Proc. IEEE Vehicular Technology Conference 2005, pp. 1758-1762, 30 May - 1 June 2005.

[22] M. Stasiak, M. Glabowski, A.Wisniewski, and P. Zwierzykowski, Modeling and Dimensioning of Mobile Networks, Wiley \& Sons, 2011.

[23] S. Hamalainen, H. Holma and A. Toskala "Capacity evaluation of a cellular CDMA uplink with multiuser detection”, Proc. of $4^{\text {th }}$ Int. Symposium on Spread Spectrum Techniques and Applications, September 1996, vol. 1, pp. 339-343.

[24] H. Akimaru and K. Kawashima, Teletraffic - Theory and Applications, $2^{\text {nd }}$ edition, Springer-Verlag, Berlin, 1999.

[25] D. Staehle, K. Leibnitz, K. Heck, B. Schröder, A. Weller, and P. Tran-Gia, “Approximating the othercell interference distribution inhomogeneous UMTS networks", Proc. IEEE Vehicular Technology Conference 2002, pp. 1640-1644, 6-9 May 2002.

[26] D. Staehle and A. Mäder, "An analytic approximation of the uplink capacity in a UMTS network with heterogeneous traffic,” Proc. 18th International Teletraffic Congress (ITC18), Berlin, pp. 81-91, 31 August 5 September 2003.

[27] Simscript III http://www.simscript.com/.(Accessed on October 2013) 